\title{
Verkehrs-, umwelt- und raumbezogene Aspekte der Elektromobilität aus der Sicht des Nutzerverhaltens
}

\author{
Thomas Döring ${ }^{1}$ Birgit Aigner-Walder ${ }^{2}$
}

Eingegangen: 14. März 2016 / Angenommen: 19. Dezember 2016 / Online publiziert: 11. Januar 2017

(C) Springer-Verlag Berlin Heidelberg 2017

Zusammenfassung Um die individuelle Akzeptanz und gesellschaftliche Verbreitung technologischer Neuerungen wie der Elektromobilität angemessen einschätzen zu können, muss die Perspektive eines potenziellen Nutzers und seines Entscheidungsverhaltens berücksichtigt werden. In Anbetracht dessen liefert der Beitrag einen Einblick in die vorliegenden Erkenntnisse zu den Bestimmungsfaktoren der subjektiven Substitutionsbereitschaft zwischen konventionellen Antriebssystemen und Elektrofahrzeugen im Bereich des motorisierten Individualverkehrs bezogen auf die damit einhergehenden Opportunitätskosten und verhaltenspsychologischen Effekte. Davon werden einige sich daraus ergebende Implikationen für den Verkehrsbereich, die Umwelt sowie die mögliche Verbreitung von Elektrofahrzeugen im urbanen und ländlichen Raum abgeleitet.

Schlüsselwörter Elektromobilität · Nutzerverhalten · Verkehr · Umwelteffekte · Urbaner Raum • Ländlicher Raum

Der Beitrag ist aus dem Arbeitskreis „Wechselwirkungen von Mobilität und Raumentwicklung im Kontext des gesellschaftlichen Wandels“ der Akademie für Raumforschung und Landesplanung heraus zunächst als Diskussionspapier entstanden (vgl. Döring/Aigner-Walder 2015). Die vorliegende Fassung stellt eine überarbeitete und zugleich gekürzte Version dieses Diskussionsbeitrags dar. Die Autoren danken sowohl den Mitgliedern des Arbeitskreises als auch den anonymen Gutachtern für ihre hilfreichen Hinweise und Kommentierungen.

This contribution has been developed within the working group "Interplay of Mobility and Spatial Development in the Context of Social Change" organized by the Academy of Spatial Research and Planning (ARL) and has been previously published in a Working Paper Series (cf. Döring/Aigner-Walder 2015). The current version, however, has been revised and abbreviated according two reviewers' suggestions during peer-review. Thus, the authors would like to thank both the reviewers for their reviews and the members of the working group for their helpful comments and suggestions.

\section{Implications of E-Mobility from a User Behavior Perspective Regarding Traffic, Environment and Space}

Abstract For the individual acceptance and social dissemination of technological innovations like e-Mobility in an appropriate manner the decision behaviour of potential users should be taken into account. In consideration of this the paper provides an overview of relevant determinants of individual willingness to substitute between conventional propulsion technologies and electrical drive engineering in the field of individual motor car traffic in terms of subjective opportunity costs and other important behaviouristic effects. Based on these insights some significant implications of e-Mobility regarding future trends in traffic, important environmental effects as well as the spread of e-Mobility in urban and rural areas will be derived.

Prof. Dr. Thomas Döring

thomas.doering@h-da.de

Prof. Dr. Birgit Aigner-Walder

b.aigner-walder@fh-kaernten.at

1 Professur für Politik und Institutionen mit Schwerpunkt Institutionenökonomik, Hochschule Darmstadt - University of Applied Sciences, Haardtring 100, 64295 Darmstadt, Deutschland

2 Professur für Volkswirtschaftslehre, Fachhochschule Kärnten, Europastraße 4, 9524 Villach, Österreich 
Keywords E-Mobility · User behaviour - Traffic · Environmental effects · Urban areas · Rural areas

\section{Einleitung und Problemstellung}

Zweifelsohne wirken sich technologische Neuerungen im Verkehrsbereich auf die Alltagsmobilität einschließlich der damit verbundenen Wahl verfügbarer Verkehrsmittel aus (vgl. für entsprechende Beispiele Bamberg 2010; Frick/ Belart/Schmied et al. 2014). Dies trifft auch auf die Entwicklung neuer Antriebstechniken im Bereich des motorisierten Individualverkehrs in Form der Elektromobilität zu. Technologische Neuerungen dieser Art bleiben dabei zum einen nicht ohne Folgen für das Nutzerverhalten. Zum anderen sind mit ihnen in aller Regel verkehrs-, umweltund raumbezogene Implikationen verbunden. In Mobilitäts- und Verkehrsstudien zeigt sich jedoch bisweilen eine vergleichsweise deterministische Sichtweise der Wirkung solcher Neuerungen dergestalt, dass von der Verfügbarkeit technologischer Möglichkeiten vorbehaltlos auf deren (zumindest mehrheitliche) Nutzung geschlossen wird (vgl. stellvertretend Kunert/Horn/Kalinowska et al. 2008; ifmo 2011). So wird dort etwa der massenhafte Anstieg der individuellen Pkw-Nutzung der zurückliegenden Jahrzehnte weitestgehend auf ein Zusammenwirken von technologischen Möglichkeiten (hier: der Option „Pkw“) und allgemeinen ökonomischen und sozialen Entwicklungstrends in modernen Industriegesellschaften zurückgeführt (vgl. hierfür stellvertretend Kutter/Stein 1998; Kutter 2001).

Bezogen auf die subjektive Akzeptanz von technischen Neuerungen sollte jedoch die Perspektive des Akteurs stärker als bislang in den Blick genommen werden, um die für Verkehr, Umwelt und Raum sich daraus ergebenden Folgen besser abschätzen zu können. Erforderlich hierfür sind Analysen, die das individuelle Entscheidungsverhalten im Umgang mit technologischen Neuerungen im Verkehrs- bzw. Mobilitätsbereich untersuchen, da allein strukturelle Änderungen noch nicht zwingend auch zu Änderungen im individuellen Verhalten führen müssen. Vielmehr sind weitere subjektive Einflussgrößen (Einstellungen, Werte, Wahrnehmung, Emotionen) dafür ausschlaggebend, ob Strukturveränderungen oder technische Innovationen auch verhaltenswirksam werden. Bezogen auf das Nutzerverhalten im Umgang mit neuen Antriebstechnologien im Bereich des motorisierten Individualverkehrs und hier speziell im Hinblick auf elektrisch betriebene Fahrzeuge hat daher die Zahl von verkehrs- und mobilitätsbezogenen Untersuchungen, die neben strukturellen auch subjektive Bestimmungsfaktoren einbeziehen, in der jüngeren Zeit stark zugenommen (vgl. Ahrend/Schwedes/Richter 2011; Peters/Hoffmann 2011; Döring/Aigner-Walder 2012; Bozem/Nagel/Rath et al. 2013; Haugrund 2013; Paternoga/
Pieper/Woisetschläger et al. 2013; Peters/Doll/Plötz et al. 2013; Pieper/Woisetschläger/Paternoga et al. 2013; Bongard 2014; Fazel 2014). Zudem ist insbesondere im Bereich der Marketingforschung die Analyse des Nutzerverhaltens von ,grünen Innovationen“ wie der Elektromobilität vermehrt in den Fokus gerückt (vgl. Hidrue/Parsons/ Kempton et al. 2011; Egbue/Long 2012; Lebeau/van Mierlo/Lebeau et al. 2012; Moons/de Pelsmacker 2012; Ziegler 2012; Steinhilber/Wells/Thankappan 2013).

$\mathrm{Zu}$ den Elektrofahrzeugen zählen dabei neben den rein batterie-elektrisch betriebenen Fahrzeugen (BEV) sowie Brennstoffzellenfahrzeugen (FCEV), welche die benötigte Energie direkt an Bord erzeugen, auch solche Fahrzeuge, die entweder über einen Verbrennungsmotor zur Reichweitenverlängerung verfügen (REEV) oder bei denen sowohl ein E-Motor als auch ein Verbrennungsmotor für den Antrieb sorgt (Plug-in-Hybridfahrzeuge - PHEV). Der Schwerpunkt der nachfolgenden Betrachtung liegt auf vierrädrigen Fahrzeugen, lediglich am Rande wird auch auf zweirädrige Fahrzeuge eingegangen. Vor diesem Hintergrund werden zunächst die vorhandenen Einsichten zum Nutzerverhalten von E-Fahrzeugen zusammenfassend dargestellt (Kapitel 2), um davon ausgehend verkehrs-, umwelt- und raumbezogene Implikationen der Elektromobilität aufzuzeigen (Kapitel 3). Der Beitrag schließt mit einem kurzen Ausblick (Kapitel 4).

\section{Elektromobilität aus Sicht potenzieller Nutzer}

Den Ausgangspunkt der nachfolgenden Überlegungen bildet die Frage, wie wahrscheinlich aus Sicht potenzieller Nutzer der Wechsel von einem konventionellen Fahrzeug zu einem Elektrofahrzeug ist, unter Berücksichtigung der damit verbundenen Opportunitätskosten (d.h. der für den Fall des Wechsels entgangenen Vorteile aus der Nutzung treibstoffgetriebener Fahrzeuge und deren charakteristischen Eigenschaften; vgl. Kapitel 2.1). Es kann dabei zwischen ökonomischen und technischen Einflussfaktoren einerseits sowie ökologischen und psychologischen Einflussfaktoren andererseits unterschieden werden.

\section{1 Ökonomische und technische Einflussfaktoren}

Während ein Vergleich der direkten Nutzungs- bzw. Betriebskosten sowie die Höhe der Anschaffungskosten einen Anhaltspunkt für die ökonomischen Opportunitätskosten von E-Fahrzeugen liefern, gelten die Reichweite und Sicherheit von E-Fahrzeugen sowie die Verfügbarkeit der benötigten Ladeinfrastruktur als wichtige technische Einflussfaktoren des individuellen Nutzerverhaltens.

Studien zeigen, dass die Energiekosten für den Gebrauch eines Elektrofahrzeugs im Durchschnitt lediglich der Hälfte 
dessen entsprechen, was an direkten Kosten für die Nutzung eines treibstoffbetriebenen Fahrzeugs zu veranschlagen ist (vgl. Döring 2012; Döring/Aigner 2011; Bertram/ Bongard 2014). Allerdings sind im Fall des E-Fahrzeugs weitere Kosten zu berïcksichtigen, die aufgrund der Batterieabnutzung entstehen und sich nach eigenen Berechnungen auf rund 7 Euro je 100 Kilometer belaufen. Bei diesem Wert werden eine Gesamtfahrleistung des E-Fahrzeugs von $150.000 \mathrm{~km}$ sowie Batteriekosten in Höhe von 10.000 Euro unterstellt (vgl. für die Batteriekosten Karle 2015: 170). Untersuchungen zeigen allerdings, dass zukünftig mit jährlich sinkenden Batteriekosten in einer Größenordnung von 6-9 \% zu rechnen ist (Nykvist/Nilsson 2015: 329). Weitere Zusatzkosten können dadurch entstehen, dass bezogen auf den aktuellen Stand der Technik eine Batterie standardmäBig nach 1.000 Ladezyklen erneuert werden muss (Karle 2015: 85). Die Zahl der maximal zu realisierenden Ladezyklen variiert dabei sowohl in Abhängigkeit vom Batterietyp (z. B. Blei-Batterie, Nickel-Metallhydrid-Batterie, Lithium-Ionen-Batterie) als auch der Außentemperatur (vgl. Wallentowitz 2013). Der erhebliche Unterschied in den direkten Nutzungskosten zwischen konventionell und elektrisch angetriebenen Fahrzeugen ist Ausdruck der divergierenden Energiedichte der verschiedenen Antriebsstoffe bzw. Speichermedien. So erreichen aktuell selbst modernste Akkus gerade einmal knapp $4 \%$ der energetischen Leistungsfähigkeit von Dieselkraftstoff (Karle 2015: 81). Die Höhe der direkten Nutzungskosten eines E-Fahrzeugs wird somit entscheidend von den Energiespeichermöglichkeiten bestimmt.

Bezüglich der Präferenzen der Nachfrager ist zudem davon auszugehen, dass die potenziellen Nutzer von E-Fahrzeugen die gleiche Qualität und Quantität des Gutes ,Mobilität" bezüglich Reichweite, zeitlicher Verfügbarkeit oder Sicherheit erwarten (vgl. Bozem/Nagel/Rath et al. 2013; Ahrend/Delatte/Kettner et al. 2014). Auch in diesem Zusammenhang stellt wiederum die Batterie eine besondere Herausforderung dar. Problematisch ist hierbei zum einen die räumliche Reichweite von reinen E-Fahrzeugen (BEV, FCEV), die bei mittlerer Geschwindigkeit gegenwärtig 150 bis 200 Kilometer beträgt, bevor die Batterie erneut aufgeladen werden muss. Im Vergleich zu einem treibstoffbetriebenen Fahrzeug (aktuelle Reichweite: 600 bis 1.000 Kilometer) weisen Elektromobile damit einen erheblichen Wettbewerbsnachteil auf (Karle 2015: 82). Hinzu kommt, dass die Reichweite eines E-Fahrzeugs zudem von topographischen und klimatischen Faktoren abhängt. Je bergiger der Streckenverlauf ist und je extremer die Außentemperaturen sind, umso niedriger ist die Reichweite (vgl. Boston Consulting Group 2010; Deutsches Clean Tech Institut 2010; Bertram/Bongard 2014). Aus der aktuell (noch) geringen Reichweite von E-Fahrzeugen (low-range usage) ergeben sich weitere Opportunitätskosten: Wollen potenziel- le Konsumenten die Gesamtheit ihrer Mobilitätsbedürfnisse in flexibler Weise befriedigen, dürfte dies häufig entweder die Anschaffung von mehr als nur einem Fahrzeug oder die zusätzliche Nutzung öffentlicher Verkehrsmittel erfordern, um auch größere Entfernungen (high-range usage) problemlos bewältigen zu können. Wenn jedoch zur jederzeitigen Befriedigung unterschiedlicher Mobilitätsbedürfnisse auf mehr als ein Verkehrsmittel zurückgegriffen werden muss, erhöht dies den Koordinationsaufwand und sorgt damit zum einen für steigende Opportunitätskosten, einen geringeren Nettonutzen und damit eine sinkende Zahlungsbereitschaft für E-Fahrzeuge. Dies heißt zum anderen aber auch, dass deren Akzeptanz in starkem Maße vom jeweiligen Haushaltseinkommen abhängt.

Hinzu kommen bislang nur unzureichend geklärte Sicherheitsfragen, bei denen aus Sicht der Opportunitätskosten wiederum die Batterietechnik ein Schlüsselfaktor ist. So stellt zum einen die Brand- und Explosionsgefahr nach wie vor eines der größten Probleme im Bereich der Speichertechnik dar. Dies gilt vor allem für die überwiegend zum Einsatz kommenden Lithium-Batterie-Varianten, die zwar allesamt vergleichsweise langlebig sind, zugleich aber auch als besonders explosiv gelten, da das hochreaktive Lithium zu Überhitzung neigt (vgl. Ziegler 2011; Hucko 2013). Inwieweit die Entwicklung neuer Batterievarianten nicht nur zu einer größeren Reichweite, sondern auch zu einem verbesserten Thermomanagement und damit zu einer höheren Nutzersicherheit führt, ist gegenwärtig noch offen (vgl. zur Weiterentwicklung der Batterietechnologie Wallentowitz 2013; Korfanty-Schiller/Ellermann/Haug et al. 2015).

Zusätzliche Opportunitätskosten verbinden sich zudem mit der Ladezeit von marktüblichen E-Fahrzeugen, die die Betankungszeit eines konventionellen Fahrzeugs um ein Vielfaches übersteigt (vgl. Pehnt/Höpfner/Merten 2007; Karle 2015). Während bei letztgenanntem Fahrzeugtyp maximal 5 Minuten ausreichen, um rund $100.000 \mathrm{kWh}$ im Tank aufzunehmen, benötigen E-Fahrzeuge 8 bis 12 Stunden für das Wiederaufladen der Batterie an der Haushaltssteckdose. Selbst im Fall einer Schnellaufladung (fast charging) beansprucht der Ladevorgang üblicherweise rund 30 Minuten (Döring/Aigner-Walder 2012: 114). Folgt man Befragungsergebnissen, wird dabei die ,Akzeptanz der Lademöglichkeiten und der damit verbundenen Ladedauer [...] bei dem Großteil der bisherigen Nutzer [...] stärker gewichtet als die Reichweite" (Ahrend/Delatte/Kettner et al. 2014: 7). Das Problem der langen Ladezeiten verweist auf das grundlegendere Problem einer zum gegenwärtigen Zeitpunkt nur unzureichend ausgebauten Infrastruktur für E-Fahrzeuge. Dies betrifft neben Werkstätten und Servicestellen vor allem die geringe Zahl öffentlich zugänglicher (Schnell-)Ladestationen. Bis zum Jahr 2020 wird allerdings mit weiterentwickelten Ladesystemen gerechnet, die deutlich höhere Spannungen und Stromstärken aufweisen 
sollen (vgl. Deutsches Clean Tech Institut 2010). Dies ist entscheidungstheoretisch insofern von Bedeutung, wie etwa das induktive Laden von E-Fahrzeugen aufgrund der damit verbundenen Zeitersparnis eine höhere Zahlungsbereitschaft bei den potenziellen Nutzern bewirkt (vgl. Papendick/Brennecke/Sánchez Márquez et al. 2011).

Die aufgrund der Batterie hohen Anschaffungskosten verhindern bislang eine breite Akzeptanz von Elektrofahrzeugen (vgl. Ahrend/Delatte/Kettner et al. 2014). So werden je nach Verbraucherstudie die Anschaffungskosten von 80-89 \% der Befragten beim Kauf eines Elektroautos als besonders wichtig eingestuft (Bozem/Nagel/Rath et al. 2013: 23; Fraunhofer IAO/PriceWaterhouseCoopers 2010: 12). Gegenwärtig liegen die Anschaffungskosten eines E-Fahrzeugs typischerweise um rund $60 \%$ (bzw. 10.000 Euro) über denen eines konventionellen Fahrzeugs (Karle 2015: 168). In den Verbraucherstudien wird darauf verwiesen, dass die Konsumenten in Deutschland jedoch lediglich einen Preisaufschlag in Höhe von etwas mehr als $20 \%$ (bzw. rund 2.500 Euro) akzeptieren würden. Auch bei einem Vergleich der gesamten Kosten des Lebenszyklus (Total Cost of Ownership), in die neben den Anschaffungsauch die laufenden Betriebs- und Werkstattkosten sowie Steueraufwendungen einfließen, liegt die Kostendifferenz zwischen elektrisch und konventionell angetriebenen Fahrzeugen immer noch bei rund 4.000 Euro (Karle 2015: 170).

\section{2 Ökologische und psychologische Einflussfaktoren}

Zusätzlich zu den bereits genannten Opportunitätskosten von E-Fahrzeugen lassen sich bezogen auf die Substitutionsentscheidung zwischen konventionell und elektrisch angetriebenen Fahrzeugen zudem ökologische und psychologische Einflussfaktoren benennen. $\mathrm{Zu}$ diesen zählt vor allem das Ausmaß an $\mathrm{CO}_{2}$-Vermeidung in Relation zu den Lebenszykluskosten von E-Fahrzeugen unter Berücksichtigung der zukünftigen Entwicklung der Energiepreise sowie spezifische verhaltenspsychologische Aspekte des Mobilitätsverhaltens.

Eine Studie der Boston Consulting Group aus dem Jahr 2009, deren Ergebnisse im Kern auch aktuell noch zutreffend sind, hat die unterschiedlichen Lebenszykluskosten verschiedener Antriebstechnologien mit geringem $\mathrm{CO}_{2}$-Ausstoß (BEV, REEV, PHEV, verbrauchsarme Verbrennungsmotoren) miteinander verglichen. Danach stellen zwar reine Elektroantriebe (BEV) jene Antriebstechnologie dar, welche die größten Mengen an $\mathrm{CO}_{2}$-Emissionen zu vermeiden in der Lage ist. Sie sind aus ökonomischer Sicht jedoch nicht die kosteneffektivste Form der $\mathrm{CO}_{2}-$ Vermeidung. Letzteres trifft vielmehr auf verbrauchsarme Verbrennungsmotoren zu, bei denen sich die Vermeidungskosten zur Reduktion von einem Prozent $\mathrm{CO}_{2}$-Emissionen bei gegebenen Energiepreisen im Vergleich zu rein batterieelektrisch betriebenen Fahrzeugen auf lediglich rund die Hälfte belaufen (vgl. Boston Consulting Group 2009). Die Studie zeigt somit, dass die umweltbezogene Kosteneffektivität von E-Fahrzeugen unterhalb der von verbrauchsarmen konventionellen Fahrzeugen liegt, solange der Ölpreis sich auf einem moderaten Niveau bewegt und erstere staatlicherseits nicht nennenswert gefördert werden.

In Anbetracht dessen überrascht nicht, dass aufgrund der momentan niedrigen Treibstoffpreise „Neuwagenkäufer in alte Verhaltensmuster" zurückfallen, was von Dudenhöffer (2015: 549) wie folgt kommentiert wird: „Mit billigem Treibstoff setzt sich der Boom der SUV in einem bisher nicht gekannten Maße fort. [...] Das zögerliche Verhalten, Elektrofahrzeuge und/oder Plug-in Hybridfahrzeuge zu kaufen, wird verstärkt. Bei Preisen konventioneller Kraftstoffe, die auf dem Niveau des Jahres 2005 liegen, verkümmert jeder Anreiz, lokal emissionslose Fahrzeuge zu kaufen." Bei aktuellen Batteriekosten in Höhe von rund 500 Euro je kWh liegen die Lebenszykluskosten eines rein batterie-elektrisch betriebenen Fahrzeugs deutlich über jenen eines Fahrzeugs mit verbrauchsarmem Verbrennungsmotor. Eine wirtschaftliche Konkurrenzfähigkeit des E-Fahrzeugs wäre demnach erst dann erreicht, wenn sich der Rohölpreis auf einem Niveau von rund 200 Euro je Barrel bewegen würde. Nur für den Fall stark sinkender Batteriekosten (z. B. auf 350 Euro je $\mathrm{kWh}$ ) ist mit einer steigenden wirtschaftlichen Attraktivität von E-Fahrzeugen zu rechnen. Dies wäre ab einem Rohölpreis in der Bandbreite zwischen 70 und 84 Euro je Barrel der Fall (Döring/Aigner-Walder 2012: 116). Zum Vergleich: Aktuell (Dezember 2016) schwankt der Rohölpreis auf dem Weltmarkt in einer Bandbreite von 47 bis 53 Euro je Barrel.

Bei den zurückliegenden Überlegungen zu den Opportunitätskosten einer privaten Nutzung von E-Fahrzeugen wurde bislang von einem weitgehend rationalen Entscheidungsverhalten ausgegangen. Zusätzlich sind hier jedoch auch Verhaltensanomalien und Verhaltensgewohnheiten infolge von subjektiven Einstellungsmustern, Werthaltungen und Wahrnehmungsverzerrungen zu berücksichtigen, wie diese etwa aus psychologischen Studien zur Verkehrsmittelwahl bekannt sind (vgl. Bamberg/Schmidt 2001; Hunecke 2006; Bamberg 2010; FehrAdvice \& Partners 2012). Danach erfordert die erstmalige Ausführung einer neuen Verhaltensweise (hier: der Kauf eines E-Fahrzeugs) eine kognitiv aufwendige, bewusste Entscheidung. Ist ein Akteur jedoch mit seinen bisher getroffenen Entscheidungen zufrieden (hier: Kauf eines konventionellen Fahrzeugs), setzt eine Gewohnheitsbildung ein, die zu Verhaltensroutinen führt (vgl. Fujii/Gärling/Kitamura 2001). Vergangene Verhaltenshäufigkeiten beeinflussen danach stark das zukünftige Entschei-

\footnotetext{
${ }^{1}$ http://www.boerse-online.de/rohstoffe/oelpreis (07.11.2016).
} 
dungsverhalten. Begünstigt wird dieses Verhaltensmuster durch verschiedene Wahrnehmungsverzerrungen wie etwa den „Status quo bias“, der den empirisch belegten Tatbestand kennzeichnet, dass Akteure eine bestehende Situation (hier: Nutzung konventioneller Fahrzeuge) möglichst nicht verändern wollen. Für die Kaufentscheidung zugunsten eines E-Fahrzeugs ist aus verhaltenspsychologischer Sicht ebenso bedeutsam, wie gut dieses zum eigenen Lebensstil, zur eigenen Persönlichkeit sowie den sonstigen sozialen Einflüssen passt. Sind E-Fahrzeuge mit den eigenen Gewohnheiten, den im Alltag zu erledigenden Aufgaben oder den wahrgenommenen situativen Handlungshemmnissen nicht kompatibel, fällt die Bereitschaft gering aus, ein solches Fahrzeug zu kaufen bzw. zu nutzen (vgl. Fraunhofer ISI 2011; Bobeth/Matthies 2016).

Damit werden Verhaltensänderungen unwahrscheinlicher, was durch die vorherrschende Berücksichtigung irreversibler Kosten (z. B. Anschaffungs- und Reparaturkosten eines im Besitz befindlichen konventionellen Fahrzeugs) noch zusätzlich begünstigt wird. Durch diese vermeintlich niedrigeren Kosten (,Sunk Cost Fallacy“) werden unter monetären Gesichtspunkten optimale Entscheidungen verhindert oder zumindest zeitlich hinausgezögert. Dies gilt auch für den „Availability Bias“, der bewirkt, dass sich Verbraucher häufiger für die leichter verfügbaren konventionellen Fahrzeuge als für die nach wie vor weniger gut verfügbaren Elektrofahrzeuge entscheiden. Begünstigt wird dies durch die gegebene Marktsituation, bei der es potenziellen Nutzern von E-Fahrzeugen (immer noch) schwer fällt, ein geeignetes Angebot zu finden, da sowohl Anzahl als auch Variationsbreite der Produkte im Vergleich zum Markt für treibstoffbetriebene Fahrzeuge nach wie vor sehr niedrig sind (vgl. Döring 2012; Dudenhöffer 2014).

\section{Verkehrs-, umwelt- und raumbezogene Implikationen der Nutzung von Elektrofahrzeugen}

Angesichts der Merkmale von E-Fahrzeugen (hohe Anschaffungskosten, stark eingeschränkte zeitliche Flexibilität der Nutzung, geringe räumliche Reichweite) ist aktuell nicht davon auszugehen, dass in absehbarer Zukunft mit einer stärkeren Nachfrage im Bereich des motorisierten Individualverkehrs zu rechnen ist. Damit rückt aber auch die von der Bundesregierung (2009: 18) für 2020 formulierte Zielgröße von einer Million Elektroautos auf deutschen Straßen sowie die bis 2030 angestrebten 5 Millionen E-Fahrzeuge in weite Ferne. Hintergrund der genannten Zielvorgaben ist bekanntermaßen zum einen das Postulat, den deutschen Automobilmarkt zu einem Leitmarkt für Elektromobilität zu machen, zum anderen wird von einer vermehrten Nutzung von E-Fahrzeugen ein nennenswerter
Beitrag des Verkehrssektors zu den klimapolitischen Vorgaben in Deutschland erwartet (vgl. Teichmann/Lehmann/ Brandt 2015).

\subsection{Verkehrsbezogene Aspekte einer verstärkten Nutzung von Elektromobilität}

Von den unterschiedlichen verkehrsbezogenen Effekten, die bei einer zukünftig vermehrten Nutzung von E-Fahrzeugen absehbar wären, soll hier vor allem auf drei mögliche Problemfelder näher eingegangen werden. Diese sind neben den erhofften Auswirkungen auf die multimodale Verkehrsmittelwahl sowie den Implikationen für die Nutzung und Finanzierung der Verkehrsinfrastruktur auch das erhöhte Unfallrisiko, das von E-Fahrzeugen für andere Verkehrsteilnehmer ausgeht.

Bezogen auf den motorisierten Individualverkehr in Deutschland gibt es aktuell keinerlei Anzeichen, dass ,reine' elektrische Antriebe (BEV, FCEV) ihrem momentanen Nischendasein entwachsen könnten (vgl. NPE 2014). So waren sowohl 2014 als auch 2015 mehr als $98 \%$ aller Pkw-Neuwagen reine Benzin- oder Dieselfahrzeuge (Dudenhöffer 2015: 549). Entsprechend sind aktuell von den insgesamt rund 45 Millionen in Deutschland zugelassenen Pkw immer noch weniger als 50.000 Fahrzeuge solche, die über einen Elektro- (BEV, FCEV) oder Hybridantrieb (REEV, PHEV) verfügen (Schwarzer/Breitinger 2016: 1). Aus entscheidungstheoretischer Sicht sind diese Zahlen keineswegs überraschend, da die prinzipielle Verfügbarkeit dieser neuen Antriebstechnologie noch keine Rückschlüsse über deren subjektive Akzeptanz und damit deren gesellschaftliche Durchsetzungsfähigkeit im Verkehrsbereich zulässt. Mit dem geringen Elektroanteil am Bestand aller Autos in Deutschland könnte sich allerdings auch die häufig kommunizierte Hoffnung nicht erfüllen, dass eine verstärkte Nutzung von E-Fahrzeugen aufgrund ihrer räumlich begrenzten Reichweite zu einer steigenden Nutzung öffentlicher Verkehrsmittel im Personennah- und -fernverkehr führt, um auf diese Weise die Vision eines ,klimaneutralen Verkehrs“ zu verwirklichen (vgl. InnoZ 2014).

Aber auch bei einer steigenden Akzeptanz von E-Fahrzeugen ist nicht zwingend davon auszugehen, dass sich der Gebrauch öffentlicher Verkehrsmittel erhöht. Vielmehr gilt eine „Kannibalisierung des Öffentlichen Verkehrs durch Elektroautos“" (Teufel/Arnold/Bauer et al. 2015: 60) als wahrscheinlich. Würden sich Elektroautos in Zukunft durchsetzen, verlieren im Hinblick auf die individuelle Mobilitätsentscheidung auch die Betriebskosten für die zu leistenden Fahrtstrecken an Gewicht, da - ausgehend von den bestehenden relativen Preisen - Strom deutlich günstiger als Benzin ist. Die mögliche Folge wäre ein Anstieg der Pkw-Nutzung bei einem Straßennetz, das bereits aktuell in vielen Regionen überlastet ist. Die Überlastung des 
Straßennetzes stellt aus ökonomischer Sicht insofern ein Problem dar, weil Deutschland durch eine Raumstruktur gekennzeichnet ist, die auf einer flächendeckend guten Versorgung mit Verkehrsinfrastruktur beruht. Letztere ist dabei nicht nur die Grundlage für eine polyzentrische Siedlungsstruktur, sondern zugleich auch Basis für die bestehende Wirtschaftsstruktur, für die eine hochgradig arbeitsteilige Vernetzung im Raum charakteristisch ist.

Neben den Auswirkungen auf den Grad der Auslastung des Straßennetzes hätte eine vermehrte Nutzung elektrisch betriebener Fahrzeuge zudem negative Folgen für die Finanzierung der Verkehrsinfrastruktur durch Bund, Länder und Kommunen (vgl. Institut der deutschen Wirtschaft 2014). Der Grund für diesen negativen Finanzierungseffekt ist, dass bei einer umfangreichen Substitution von konventionell angetriebenen Fahrzeugen durch Elektrofahrzeuge die bisherigen Finanzierungsquellen für den Verkehr wegzufallen drohen, da für diesen Fall mit einem starken Rückgang der Einnahmen aus der Mineralölsteuer gerechnet werden muss (vgl. Maaß 2014). Dem stünden volkswirtschaftlich dann zwar auch verminderte Aufwendungen für Ölimporte gegenüber. Diese Minderaufwendungen bei den Importen hätten jedoch keinerlei kompensatorische Wirkung bezogen auf die Einnahmeausfälle im Bereich der öffentlichen Haushalte und die damit verbundene fiskalische Lücke hinsichtlich der Verkehrsinfrastrukturfinanzierung, die durch Mehreinnahmen an anderer Stelle gedeckt werden müsste. Ein Konzept für die Anlastung der Straßeninfrastrukturkosten bei E-Fahrzeugen fehlt jedoch bislang. Da eine solche Anlastung der Verkehrsinfrastrukturkosten ,,über den Strompreis nicht möglich ist, wäre es wahrscheinlich nur über eine fahrleistungsabhängige $\mathrm{Pkw}-$ Maut entsprechend der Lkw-Maut realisierbar“ (Teufel/Arnold/Bauer et al. 2015: 60).

$\mathrm{Zu}$ den verkehrsbezogenen Effekten ist schließlich auch das erhöhte Unfallrisiko für Fußgänger und Fahrradfahrer $\mathrm{zu}$ rechnen, welches nach bisherigen empirischen Befunden mit einer verstärkten Nutzung von E-Fahrzeugen im Vergleich zu konventionell angetriebenen Fahrzeugen einhergeht (vgl. Donath 2014). So belegen Daten aus den USA, dass die durch E-Fahrzeuge ausgelöste Zahl an Fußgängerunfällen um $44 \%$ höher ist, während der entsprechende Wert für Fahrradunfälle sogar $72 \%$ beträgt (US-Department of Transportation - National Highway Traffic Safety Administration 2009: 12 ff.). Der Grund für die höhere Gefährdung von Fußgängern und Fahrradfahrern durch reine Elektro- (BEV, FCEV) ebenso wie Hybridfahrzeuge (REEV, PHEV) ist, dass diese im Stadtverkehr - insbesondere bei Geschwindigkeiten unter $50 \mathrm{~km} / \mathrm{h}$ - aufgrund fast nicht vorhandener Motorengeräusche kaum oder gar nicht hörbar sind. Im Verkehrsgeschehen orientieren sich Fußgänger und Fahrradfahrer neben optischen auch mittels akustischer Reize, um herannahende Fahrzeuge zu erken- nen. Zwar haben die Autohersteller mittlerweile durch den Einbau eines Sound-Generators auf dieses Problem reagiert, dieser zählt jedoch bislang nur in den USA und Japan aufgrund von gesetzlichen Vorgaben zur serienmäßigen Ausstattung von E-Fahrzeugen (vgl. Teufel/Arnold/Bauer et al. 2015), auch wenn dadurch der Vorteil geringer Lärmemissionen zunichte gemacht wird.

\subsection{Umweltbezogene Effekte einer verstärkten Nutzung von Elektromobilität}

Unter Umweltaspekten wird von der Elektromobilität ein nennenswerter Beitrag zur nachhaltigen Einsparung von $\mathrm{CO}_{2}$-Emissionen im Verkehrsbereich erwartet, was zugleich als ein positiver Anreiz für den Kauf eines E-Fahrzeuges gilt. Eine ökologische Bewertung setzt allerdings eine vergleichende Betrachtung der gesamten Umweltbilanz von E-Fahrzeugen voraus, was neben den ökologischen Effekten der direkten Nutzung auch jene der Herstellung, der Entsorgung sowie der Energiegewinnung mit einschließt. So gelten E-Fahrzeuge hinsichtlich ihres Energieverbrauchs zwar im Vergleich zu benzin- oder dieselbetriebenen Fahrzeugen als deutlich effizienter, da sie - abhängig vom Fahrzeugtyp - nur die Hälfte oder gar ein Viertel der Energie für das Zurücklegen derselben Wegstrecke benötigen (vgl. Degelmann 2014). Positive Umweltwirkung und Wirtschaftlichkeit (hier: effizienzbedingte Kosteneinsparung) sind insofern eng miteinander verknüpft. Hinzu kommt, dass während des Betriebs von E-Fahrzeugen nur geringe Lärmbelästigungen, keine Feinstaubemissionen sowie keine $\mathrm{CO}_{2}$ - oder $\mathrm{NO}_{\mathrm{x}}$-Abgase entstehen (vgl. Deutsches Clean Tech Institut 2010). Ein eindeutiger Vorteil von E-Fahrzeugen ist jedoch nur dann gegeben, wenn die benötigte Energie überwiegend aus regenerativen Energiequellen gewonnen wird. Würde in Deutschland der politische Zielwert von einer Million Elektroautos bis 2020 realisiert, so wäre damit ein Energiebedarf von rund 1,98 Mrd. kWh verbunden, was etwa 0,3\% des deutschen Stromverbrauchs in 2015 entspricht. Geht man dabei von einem Bruttostromverbrauch aus, der $\mathrm{zu} 30 \%$ aus regenerativen Energien gewonnen wird, könnten eine Million E-Fahrzeuge schätzungsweise 1,14 Mrd. Tonnen $\mathrm{CO}_{2}$ vermeiden. Bei einem Anteil von $50 \%$ erneuerbaren Energien ließe sich der bestehende $\mathrm{CO}_{2}$-Ausstoß sogar um rund 1,88 Mrd. Tonnen reduzieren (Deutsches Clean Tech Institut 2010: 38; Döring/Aigner-Walder 2012: 106).

Betrachtet man allerdings den gesamten Lebenszyklus von E-Fahrzeugen, ist dieser mit einem hohen Energiebedarf verbunden. Dies gilt nicht allein für die Fertigung der Batterien. Vielmehr muss hier die gesamte Wertschöpfungskette in den Blick genommen werden, zu der auch die Extraktion der benötigten Rohstoffe (neben Lithium etwa ebenso Silizium), deren Transport und spätere Entsorgung bzw. Wiedergewinnung zu rechnen sind (vgl. 


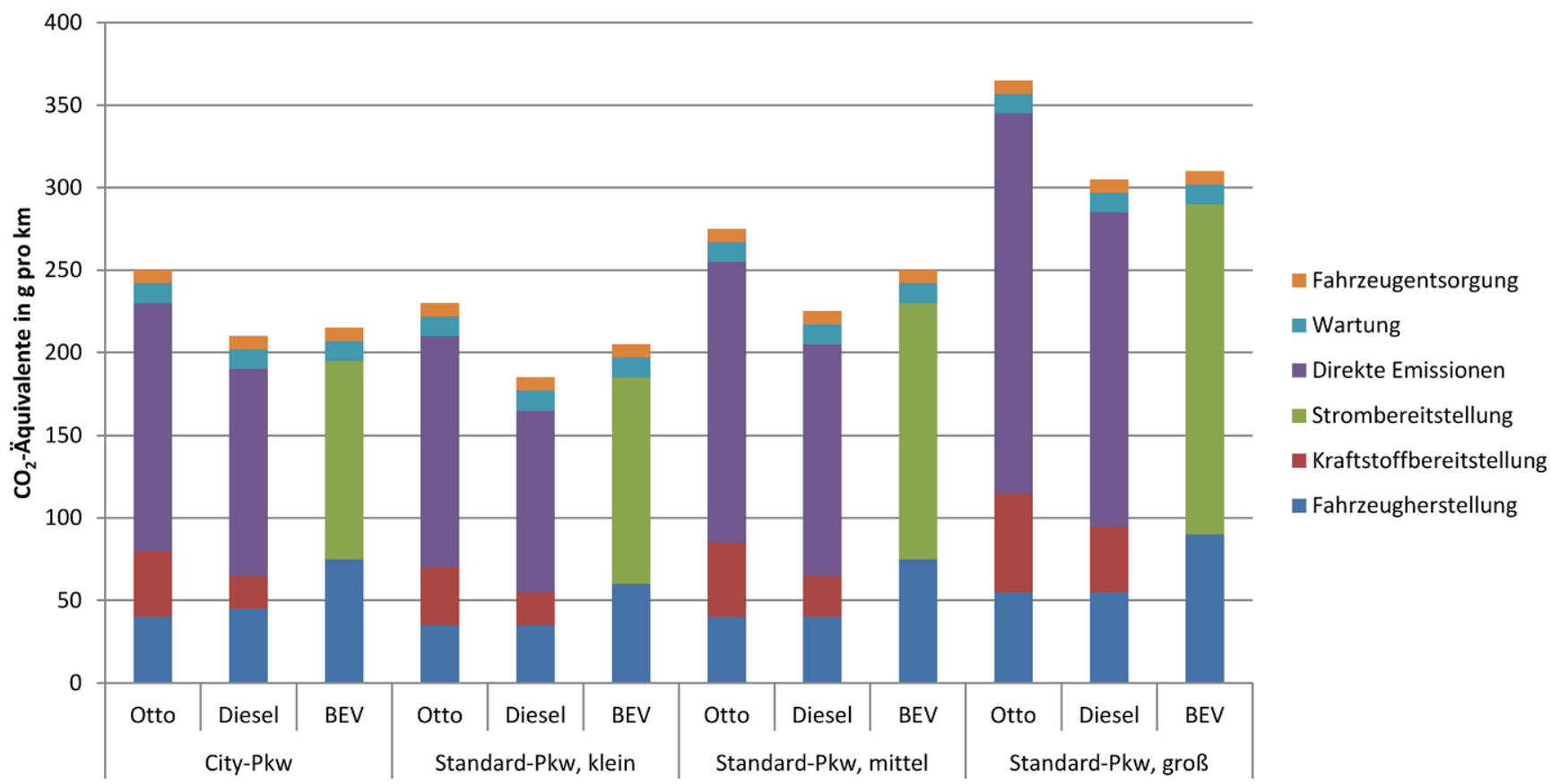

Abbildung 1 Vergleich der $\mathrm{CO}_{2}$ - ̈̈quivalente verschiedener Fahrzeugtypen mit unterschiedlicher Antriebstechnologie (in g pro km) Quelle: Eigene Darstellung auf der Grundlage von Degelmann (2014: 25) und ifeu (2011: 17)

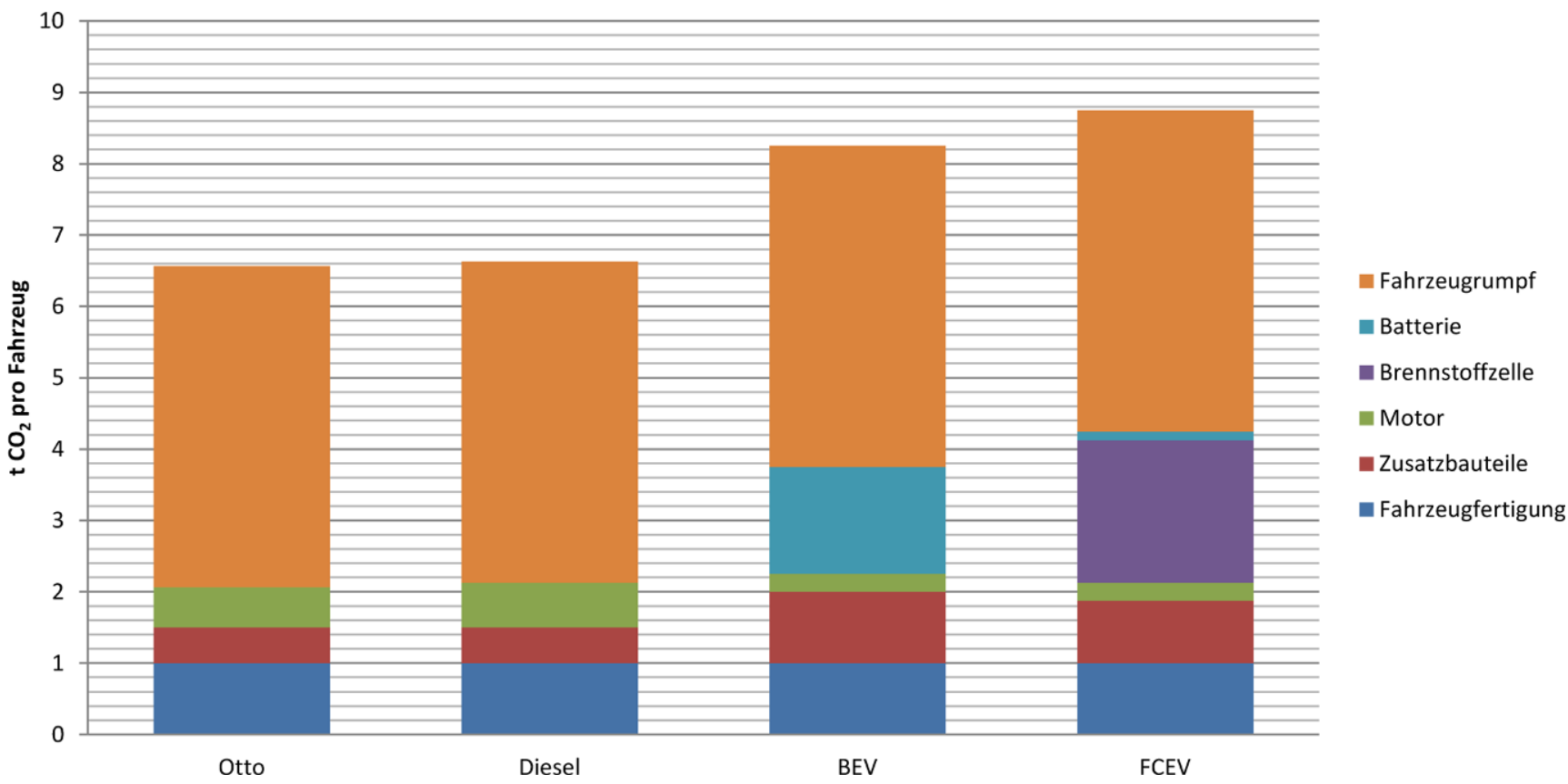

Abbildung 2 Vergleich der Klimawirkung der Herstellung eines mittleren Pkw mit unterschiedlicher Antriebstechnologie (in $\mathrm{C} \mathrm{CO}_{2}$ pro Fahrzeug) Quelle: Eigene Darstellung auf der Grundlage von Degelmann (2014: 25) und ifeu (2011: 20)

Pehnt/Höpfner/Merten 2007; ifeu 2011; Döring/AignerWalder 2012). Hinzu kommt, dass mit der Batterie eines E-Fahrzeugs aufgrund der darin enthaltenen Schwermetalle ein erhebliches Umweltrisiko verbunden ist. Aber auch wenn der Blick allein auf die Treibhausgasemissionen (gemessen in $\mathrm{CO}_{2}$ - Äquivalenten) pro Kilometer gerichtet ist, die mit der Fahrzeugherstellung, der Kraft-
stoff-/Strombereitstellung, dem unmittelbaren Betrieb, der Wartung sowie der Fahrzeugentsorgung verbunden sind, zeigt eine vergleichende Klimabilanz von konventionell und elektrisch betriebenen Fahrzeugen, dass im Fall des bestehenden Anteils an regenerativen Energien an der deutschen Stromherstellung E-Fahrzeuge zwar im Vergleich zu benzinbetriebenen Fahrzeugen günstigere Werte aufwei- 


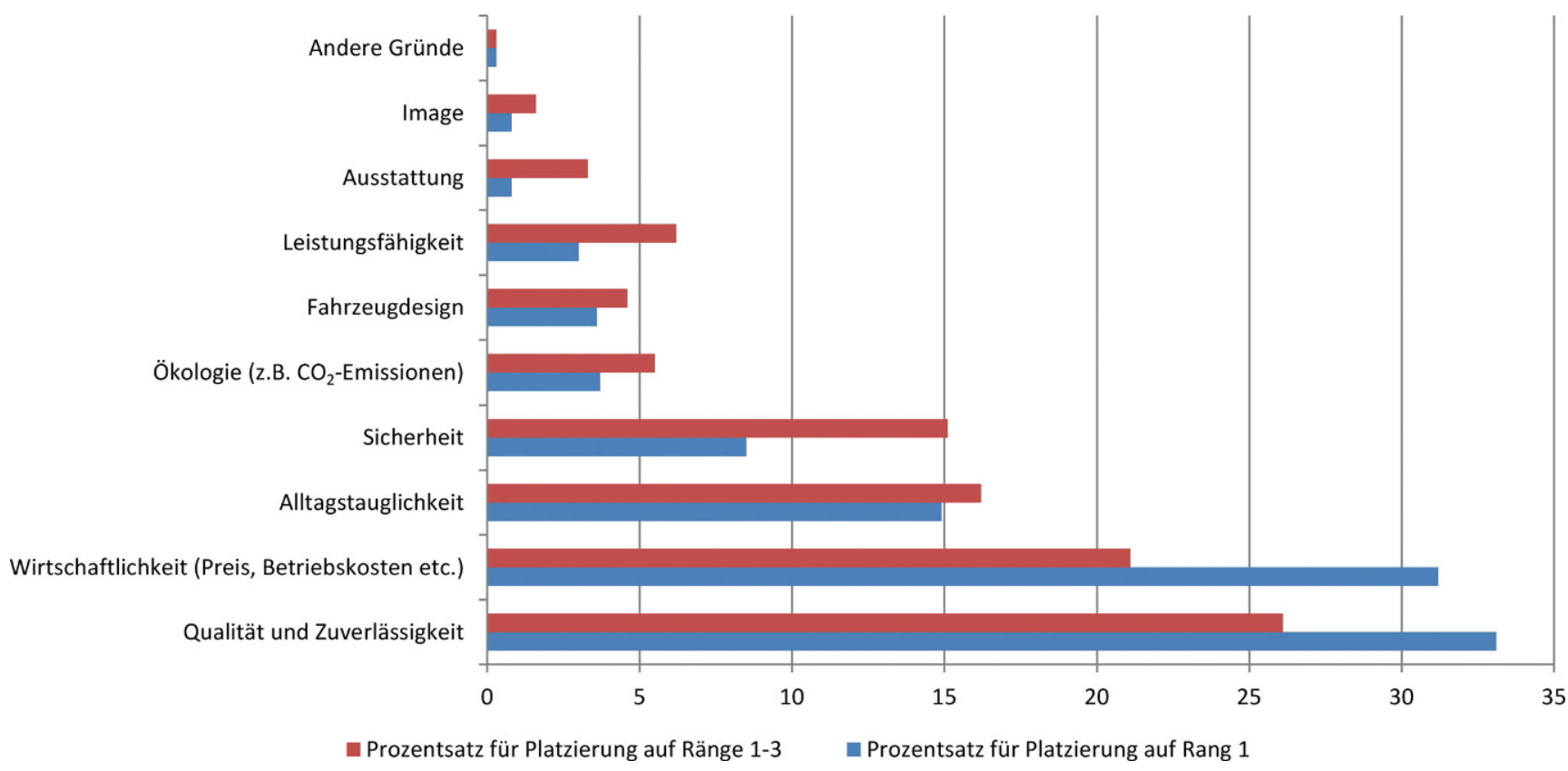

Abbildung 3 Bestimmungsfaktoren des Kaufs von Elektrofahrzeugen (Zustimmung der Befragten in \%) Quelle: Eigene Darstellung auf der Grundlage von Bongard (2014: 6)

sen, nicht jedoch gegenüber dieselbetriebenen Fahrzeugen (vgl. Abbildung 1). Betrachtet man allein den Herstellungsprozess, fällt auch die Klimawirkung von benzinbetriebenen Fahrzeugen günstiger aus (vgl. Abbildung 2). Der Einteilung der untersuchten Fahrzeuge in City-Pkw bzw. Standard-Pkw unterschiedlicher Größe (klein, mittel, groß) liegen dabei „keine spezifischen Fahrzeugmodelle, sondern generalisierte, mittlere Fahrzeugtypen nach heutigem Stand der Technik" zugrunde (ifeu 2011: 14). Zudem werden FCEV-Fahrzeuge lediglich als ein Unterfall von BEV-Fahrzeugen angesehen und daher nicht gesondert in die vergleichende Betrachtung mit einbezogen (Degelmann 2014: 24).

Bezüglich der Klimawirkung unterscheiden sich die verschiedenen Antriebstechniken bezogen auf Herstellung und Entsorgung des Fahrzeugs nur geringfügig. Dies gilt in gleicher Weise für jene Schadstoffe, die bei der Förderung und Erzeugung von Benzin oder Diesel einerseits sowie der Produktion einer Lithium-Ionen-Batterie für ein E-Fahrzeug andererseits anfallen. Muss Letztere allerdings vor dem Ablauf der Lebenszeit eines E-Fahrzeugs ausgetauscht werden, verschlechtert sich dessen Gesamtbilanz deutlich (vgl. Bertram/Bongard 2014; Asendorpf 2015). Rund zwei Drittel der Emissionen entstehen beim Betrieb eines Fahrzeugs, wobei der $\mathrm{CO}_{2}$-Ausstoß eines E-Fahrzeugs etwas geringer als bei einem Dieselfahrzeug und deutlich geringer als bei einem Benzinfahrzeug ausfällt. Demgegenüber schneiden E-Fahrzeuge, die im deutschen Stromnetz betankt werden, bei den Schadstoffen aus Feinstaub, radioaktivem Abfall, Schwefel- und Stickoxiden im Vergleich zu konventionel- len Antriebstechniken deutlich schlechter ab. Diese entstehen vor allem in den Kraftwerken, beim Feinstaub aber auch durch die Förderung und Aufbereitung von Kupfer, Nickel und Lithium für die Batterien. Zudem ist zu berücksichtigen, dass durch die Herstellung von Flüssigkraftstoffen auf der Basis von biologischen Rohstoffen, durch Rückhaltetechniken sowie durch Fortschritte bei der Reduzierung des Energieverbrauchs die Klimabilanz konventionell angetriebener Fahrzeuge noch weiter verbessert werden kann (vgl. Canzler/Knie 2015). Allerdings wird diese positive Umweltwirkung konventioneller Antriebstechniken zum einen durch Rebound-Effekte aufgrund einer steigenden Nachfrage nach Fahrzeugklassen mit größerer Motorleistung eingeschränkt (vgl. Dudenhöffer 2014). Zum anderen können Emissionseinsparungen bei konventionell betriebenen Fahrzeugen nur unter deutlich höheren Grenzkosten im Vergleich zu E-Fahrzeugen realisiert werden (vgl. ifeu 2011).

Ob diese umweltbezogenen Aspekte aus Sicht des Nutzerverhaltens von besonderer Bedeutung sind, muss allerdings als fraglich gelten. So kommt eine aktuelle Untersuchung zum Ergebnis, dass lediglich ein geringer Prozentsatz (3,7 \% der Befragten) bei der Reihung von ,Kauffaktoren “ den ökologischen Vorteil alternativer Antriebstechnologien als primäre Einflussgröße (Rang 1) benennt (Bongard 2014: 6; vgl. Abbildung 3). Der Wert erhöht sich leicht auf knapp $5 \%$, wenn man auch jene Befragten berücksichtigt, die den ökologischen Vorteil unter den ersten drei Rängen platzieren. Von größerer Relevanz sind aus Sicht der Nutzer demgegenüber Faktoren wie die Qualität und Zuverlässigkeit von E-Fahrzeugen $(33,1 \%)$, deren Wirt- 


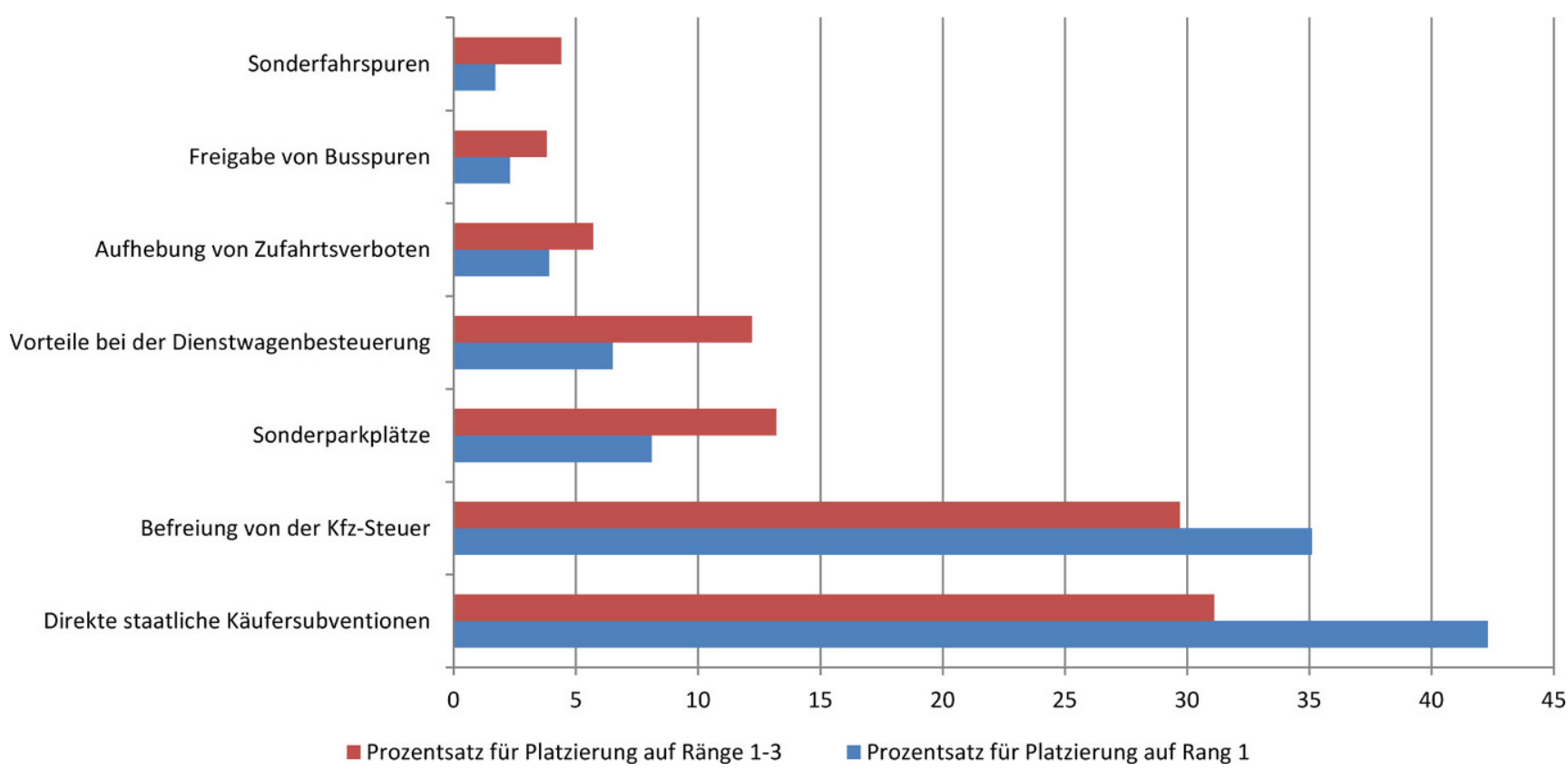

Abbildung 4 Präferenz von Fördermaßnahmen zugunsten von Elektromobilität (Zustimmung der Befragten in \%) Quelle: Eigene Darstellung auf der Grundlage von Bongard (2014: 5)

schaftlichkeit (31,2\%), Alltagstauglichkeit $(14,9 \%)$ oder auch Sicherheit $(8,5 \%)$. Im Vergleich dazu spielen stärker psychologisch bedeutsame Determinanten wie etwa das Design $(3,0 \%)$ oder das Image $(0,8 \%)$ von Elektrofahrzeugen eine ähnliche geringe Rolle wie deren ökologische Effekte (Bongard 2014: 6). Die Prozentwerte für die beiden erstgenannten Kauffaktoren sinken leicht, wenn man kumulativ auf die Platzierung der einzelnen Faktoren auf den ersten drei Rängen abstellt. Dieses auf den ersten Blick überraschende Ergebnis lässt sich damit erklären, dass die beiden Betrachtungsweisen (nur Rang 1 vs. Ränge 1-3) zu unterschiedlichen Häufigkeitsverteilungen führen. Eine alleinige Betrachtung der Prozentsätze für die Platzierung auf Rang 1 würde nur eingeschränkt aussagekräftig sein, da die Kaufentscheidung eines potenziellen Nutzers in aller Regel einen multifaktoriellen Charakter aufweist.

Das hohe Gewicht ökonomischer Faktoren für die Kaufentscheidung zeigt sich im Rahmen der genannten Untersuchung darüber hinaus im Hinblick auf die Frage nach den wünschenswerten Fördermaßnahmen für E-Fahrzeuge (vgl. Abbildung 4). Danach präferieren 42,3\% der befragten Personen staatliche Subventionen im Fall des Kaufs entsprechender Fahrzeuge als wichtigste Maßnahme, gefolgt von $35,1 \%$ der Nennungen, die für eine Befreiung der E-Fahrzeuge von der Kfz-Steuer zwecks finanzieller Entlastung votieren (Bongard 2014: 5). Im Vergleich dazu wird solchen Fördermaßnahmen, die in der aktuellen politischen Diskussion häufig genannt werden, wie die Einrichtung von Sonderparkplätzen für E-Fahrzeuge $(8,1 \%)$, etwaige finanzielle Vorteile bei der Dienstwagenbesteuerung von Unterneh- men $(6,5 \%)$, die Aufhebung von Zufahrtsverboten (3,9\%), die Freigabe von Busspuren im städtischen Raum (2,3 \%) oder auch die Einrichtung von Sonderfahrspuren (1,7\%), nur eine vergleichsweise geringe Bedeutung bezüglich der Kaufentscheidung beigemessen (Bongard 2014: 5). Auch hier verändern sich die Häufigkeitsverteilung der Befragungsergebnisse und damit die Prozentwerte der einzelnen Fördermaßnahmen, wenn man die Nennungen zu den ersten drei Rängen kumulativ auswertet. Zumindest bezogen auf die Subventionierung von E-Fahrzeugen hat die Bundesregierung gemeinsam mit der Industrie eine entsprechende Kaufprämie, die offiziell als „Umweltbonus“ bezeichnet wird, mit Wirksamkeit seit Anfang Juli 2016 beschlossen (vgl. Schwarzer/Breitinger 2016).

\subsection{Räumliche Aspekte der Nutzung von Elektrofahrzeugen}

Die Analyse bliebe unvollständig, wenn nicht auch auf räumliche Unterschiede bezogen auf die Nutzung von E-Fahrzeugen eingegangen würde. Vor allem von Bedeutung sind hierbei solche Verhaltensunterschiede, die sich bei einer Differenzierung zwischen urbanen und ländlichen Räumen zeigen.

\subsubsection{Elektromobilität im städtischen und stadtnahen Raum}

Ein Vorteil von Agglomerationsräumen ist vor allem, dass ein bislang wesentlicher Nachteil der Elektromobilität 


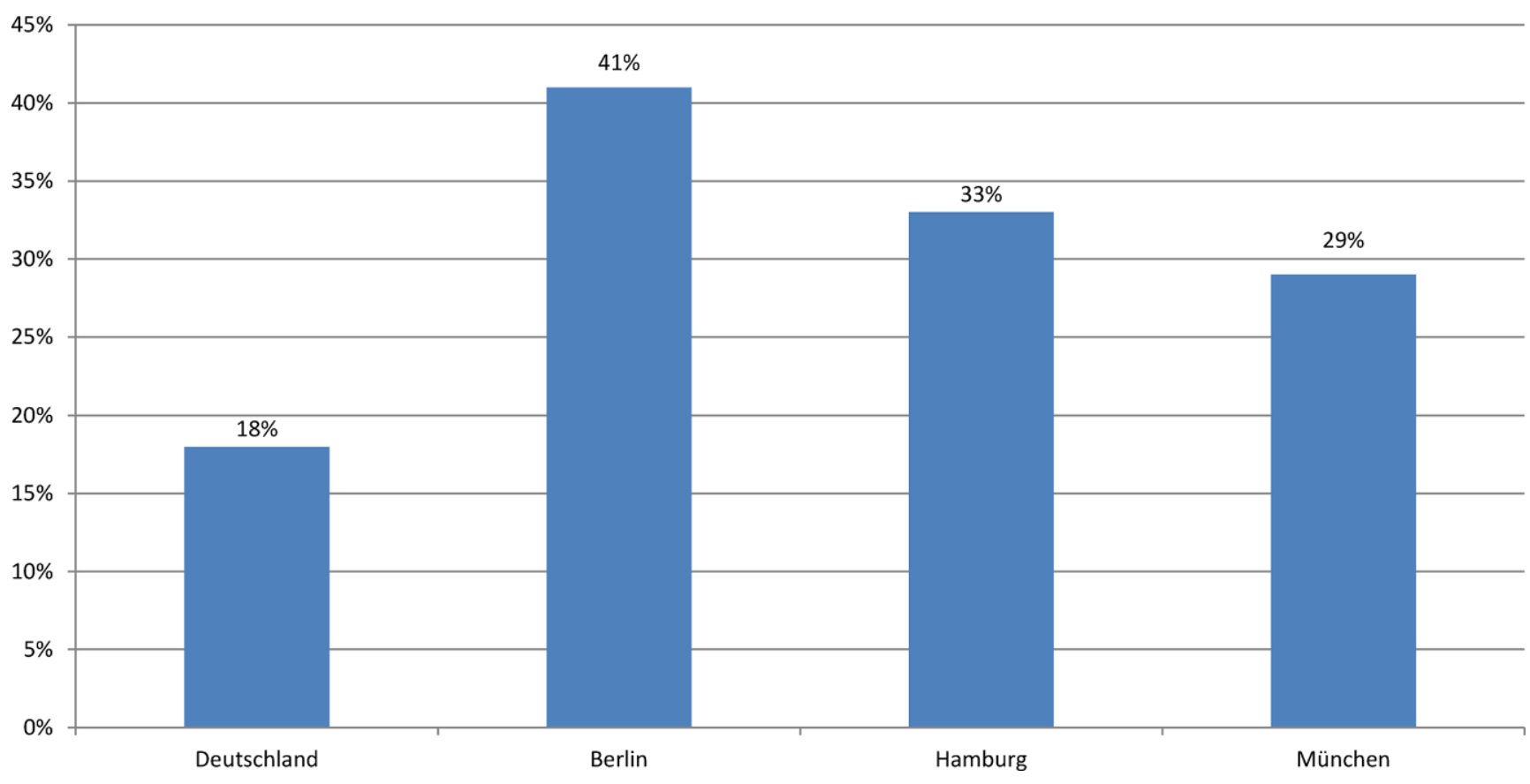

Abbildung 5 Anteil autofreier Haushalte in Deutschland sowie in ausgewählten Großstädten (in \%) Quelle: Eigene Darstellung auf der Grundlage von Canzler/Knie (2015: 24)

in Gestalt der geringen Reichweite im städtischen bzw. stadtnahen Raum keine oder eine lediglich untergeordnete Rolle bezogen auf die individuelle Substitutionsbereitschaft spielt. Dies trifft sowohl auf die rein private Nutzung von E-Fahrzeugen als auch den Einsatz solcher Fahrzeuge im städtischen Lieferverkehr zu. So müssen Fahrzeuge im Auslieferungsdienst im Durchschnitt tägliche Strecken von 100 bis 120 Kilometer zurücklegen, was mit reinen Elektrofahrzeugen (BEV) im Regelfall ohne Zwischenspeicherung möglich ist (vgl. Canzler/Knie 2015; Gries/Zelewski 2015). Zudem eignen sich die städtischen Verdichtungsräume aus Nutzersicht nicht nur für einen verstärkten Gebrauch von Elektroautos, sondern auch von E-Fahrrädern und E-Motorrädern. Vor allem bei den E-Fahrrädern (Pedelecs) zeigt sich eine steigende Nachfrage sowie eine steigende Zahl von Neuverkäufen. Die eigentliche ,Innovation der Elektromobilität" scheint sich angesichts dessen im Bereich der zweirädrigen E-Fahrzeuge abzuzeichnen (vgl. Löfken 2010). Aus der Perspektive des Nutzerverhaltens ist dies keineswegs überraschend: „Im Gegensatz zum Elektroauto, das im Vergleich zum herkömmlichen Auto als mobilitätseinschränkend erlebet wird, wird das Pedelec als ein Zugewinn zur persönlichen Mobilität wahrgenommen“ (Ahrend/Delatte/Kettner et al. 2014: 6).

Die Nutzung von E-Fahrrädern trägt nicht unerheblich zur Erweiterung des räumlichen Aktionsradius insbesondere der älteren Bevölkerungsgruppen bei - und dies sowohl im urbanen wie auch im ländlichen Raum. Richtet man den Blick vor allem auf diese Variante der Elektromobilität, dann ,kann sie zur Vielfalt auf der Straße beitragen, die im Sinne anlassbezogener Verkehrsmittelnutzung und multimodaler Fortbewegung erwünscht ist" (Degelmann 2014: 24). Begünstigt wird diese Entwicklung nicht nur dadurch, dass in urbanen Räumen vermehrt jene Bevölkerungsgruppen anzutreffen sind, die sowohl hinsichtlich ihrer ökologischen Präferenzen als auch aufgrund ihres Pro-Kopf-Einkommens über die notwendige Zahlungsbereitschaft und Zahlungsfähigkeit für den Kauf von E-Fahrzeugen verfügen. Ebenfalls von Bedeutung ist, dass insbesondere in den Agglomerationsräumen die Ansprüche, ein eigenes Auto besitzen zu müssen, merklich zugunsten einer intermodalen Verkehrspraxis in den zurückliegenden Jahren zurückgegangen sind. Als ein Beleg hierfür kann angesehen werden, dass der Anteil autofreier Haushalte in Großstädten wie Berlin, Hamburg oder auch München mit Werten zwischen 30 und $40 \%$ erheblich über dem Durchschnittswert von $18 \%$ für Deutschland insgesamt liegt (vgl. Abbildung 5).

Auch kann festgestellt werden, dass sich vor allem in den Großstädten das Mobilitätsverhalten im Hinblick auf die Verkehrsmittelwahl in Richtung Multimodalität verschoben hat (vgl. InnoZ 2014). Damit erfolgt insbesondere in den Städten die Befriedigung bestehender Mobilitätsbedürfnisse aus individueller Sicht nicht mehr allein unter Rückgriff auf ein Hauptverkehrsmittel (hier: das Auto). Vielmehr gewinnt anlassbezogen sowie je nach sachlicher Verfügbarkeit und räumlicher Zugänglichkeit die Kombination unterschiedlicher Verkehrsmittel (Auto, Busse, Bahnen, Fahrrad) zunehmend an Bedeutung (vgl. Abbildung 6). Die Akteure in großen Städten - Bewohner wie Besucher - bewegen sich damit verkehrsbezogen zunehmend vernetzt bzw. intermo- 


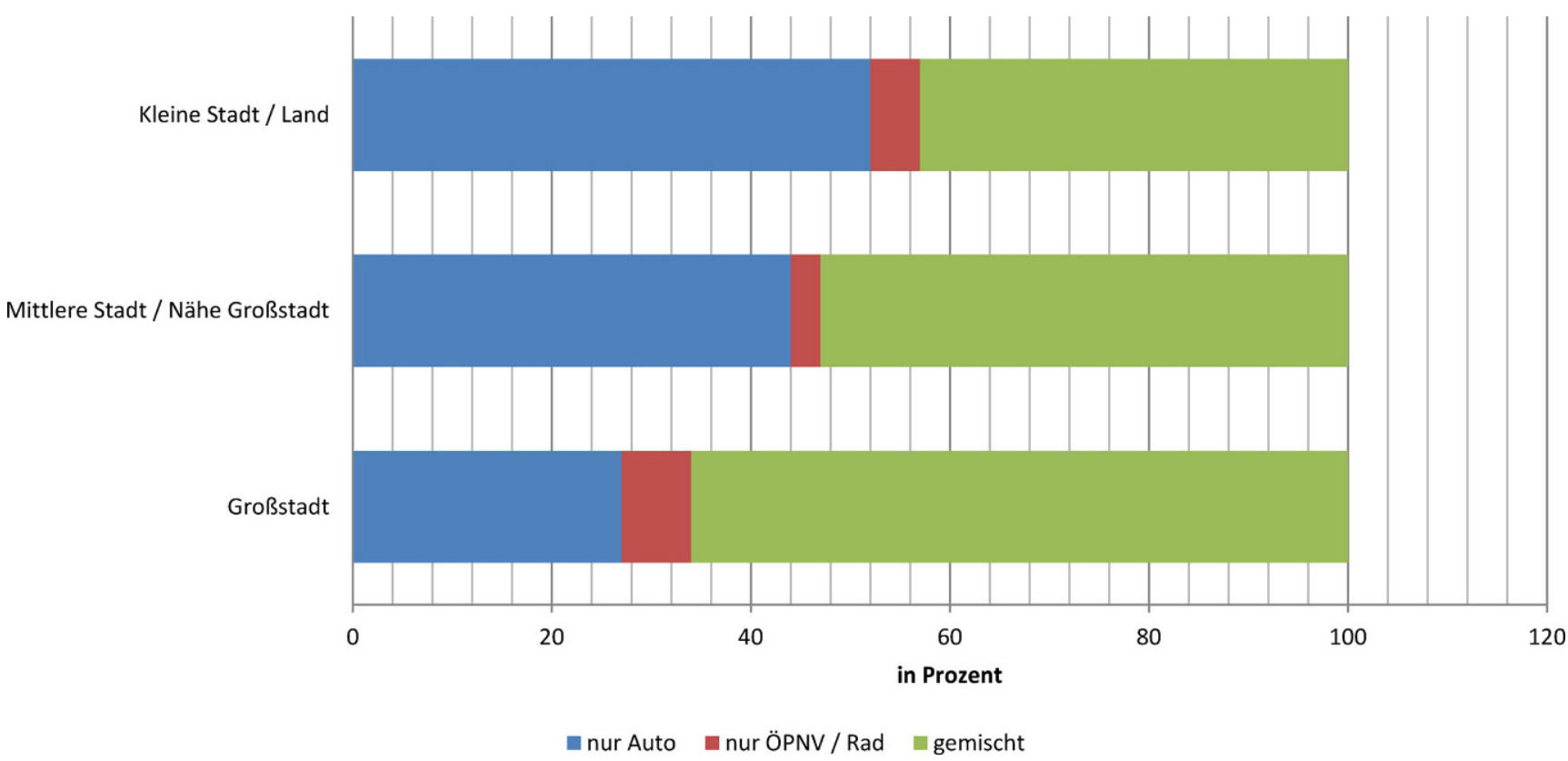

Abbildung 6 Verkehrsmittelnutzung in Deutschland nach Regionen (in \%) Quelle: Eigene Darstellung auf der Grundlage von Canzler/Knie (2015: 24)

dal. Demgegenüber ist in mittleren wie kleineren Städten ebenso wie im ländlichen Raum die individuelle Verkehrsmittelwahl in deutlich stärkerem Maße durch die Nutzung des Autos geprägt.

Damit im Einklang stehen die Ergebnisse neuerer Untersuchungen, die zeigen, dass vor allem die Mitglieder der jüngeren Alterskohorten in den Städten zwar nach wie vor den Führerschein erwerben, diese jedoch im Vergleich zu ihren Altersgenossen im ländlichen Raum deutlich weniger mit dem Auto fahren (Kuhnimhof/Armoogum/Buehler et al. 2012; Kuhnimhof/Buehler/Wirtz et al. 2012; Schönduwe/Bock/Deibel 2012; Delbosc/Currie 2014; McDonald 2015). Demgegenüber gewinnt die Nutzung von Bussen und Bahnen, insbesondere jedoch von Fahrrädern, zunehmend an Bedeutung. Soweit Autos als Fortbewegungsmittel genutzt werden, gewinnen wiederum privates Autoteilen und gewerbliches Carsharing stetig an Relevanz (vgl. zum „Peak-Car-Phänomen“ etwa Kuhnimhof/Zumkeller/Chlond 2013; Schreckenberg 2015; van Wee 2015). Damit verbunden ist eine Digitalisierung des Alltagslebens, bei der sich für an Smartphones gewöhnte urbane Akteure die Handhabung des Carsharing, aber auch die Nutzung des ÖPNV vergleichsweise einfach gestaltet (vgl. Bratzel 2011; Canzler/Knie 2015; Konrad/Wittowsky 2016).

Aus Sicht der Nutzerakzeptanz von Elektromobilität sind die genannten Verhaltenstrends insofern bedeutsam, als ein nutzerfreundlicher Gebrauch von E-Fahrzeugen vor allem im Rahmen von Fahrzeugflotten gewährleistet werden kann. Zugleich begünstigt dies sogenannte „Hub-and-Spoke"-Konzepte: Durch wohn- und arbeitsortnahe Standorte von $\mathrm{zu}$ buchenden E-Fahrzeugen wird der städtisch-klein- räumige Verkehr individualisiert unternommen (Spoke; Speiche), während längere Strecken (Hubs; Naben) mit fließendem Übergang auf der Schiene absolviert werden. In dieser multimodalen Kombination verlieren bisherige Hemmnisse einer vermehrten Nutzung von E-Fahrzeugen (lange Batterieladezeiten, geringe Reichweite) vor allem im städtischen Raum erheblich an Gewicht. Diese erwünschte Multimodalität ist allerdings nicht selbstdurchsetzend, sondern sollte vor allem auf der kommunalen Ebene durch entsprechende Fördermaßnahmen anreizkompatibel gestaltet werden. Zudem setzt die Kombination von E-Fahrzeugen mit öffentlichen Verkehrsmitteln hinreichend attraktive Angebote im Bereich des öffentlichen Personennahverkehrs voraus (vgl. Ahrend/Delatte/Kettner et al. 2014).

Ein verstärkter Einsatz von E-Fahrzeugen im Rahmen von gewerblichen Fahrzeugflotten (Carsharing, Taxiunternehmen, Logistikunternehmen etc.; vgl. auch Hacker/von Waldenfels/Mottschall 2015), wie dies vor allem im urbanen Raum möglich ist, könnte zudem einen Beitrag zur Energiewende leisten, wenn man zusätzlich zur Mobilitätsfunktion auch die Energiespeicherfunktion der Fahrzeuge (vehicle-to-grid - V2G) nutzt. Dabei können mit dem Energienetz verbundene E-Fahrzeuge, die sich im Modus des gesteuerten (das heißt innerhalb einer definierten Periode zeitversetzten) Ladens befinden, bekanntermaßen als Puffer fungieren (vgl. Karle 2015). Ein umfassendes Ausfüllen dieser Pufferfunktion setzt allerdings ein bidirektionales Laden voraus, das heißt, es erfolgt bei Bedarf nicht nur eine Einspeisung an überschüssiger Energie in die Fahrzeugbatterie, sondern es kommt bei Bedarf auch zu einer Rückspeisung ins Verteilernetz. Ein dergestalt flexibler Rückgriff 
auf die Batterie ist allerdings mit Verfügbarkeitseinschränkungen in der Fahrzeugnutzung verbunden, die sich wiederum negativ auf die individuelle Nutzerakzeptanz auswirken können.

Eine potenzielle Zielgruppe für ein gesteuertes Laden im Bereich der privaten Haushalte sind noch am ehesten Pendler in Agglomerationsräumen, die auf Betriebsparkplätzen ihr Fahrzeug an das Stromnetz anschließen und auf diese Weise mit zur Absorption der Mittagsspitzen, die bei der Gewinnung von Energie aus regenerativen Quellen auftreten, beitragen. Von größerer Bedeutung ist hier jedoch der unternehmerische Flottenbetrieb, wie er sich in einem quantitativ hinreichenden Umfang vorrangig im urbanen Raum findet. Eine in diesem Zusammenhang wichtige Einflussgröße dürfte sein, in welchem Maße durch differenzierte Tarife, Einspeisungsvergütungen und Netzstabilisierungsboni die individuellen Anreize so verändert werden können, dass das Interesse an der Nutzung von E-Fahrzeugen steigt (vgl. Fraunhofer IWES 2013; Jansen/Richts/Gerhardt et al. 2015; Sterner/Eckert/Thema et al. 2015). Ob sich dieses Szenario einer vorrangig im städtischen Raum praktizierten Verschränkung von Energiewende und Elektromobilität umsetzen lässt, hängt neben individuellen Vorteilsabwägungen aber auch von technologischen Restriktionen ab: So stehen und fallen V2G-Modelle ,letztlich mit der technischen Fähigkeit der Speichereinheiten im Fahrzeug, präzise und zuverlässig speichern und rückspeisen zu können“" (Canzler/Knie 2015: 31).

Für eine vorrangige Nutzung von E-Fahrzeugen im urbanen Raum spricht zudem, dass in Ballungsräumen die Voraussetzungen günstiger sind als im ländlichen Raum, um den flächendeckenden Ausbau der benötigten Ladeinfrastruktur mit öffentlichem oder halböffentlichem Zugang voranzutreiben. Die Voraussetzung hierfür liefern wiederum technische Innovationen, wie etwa die vergleichsweise kostengünstige Nutzung des vorhandenen Straßenbeleuchtungsnetzes als Stromzapfstellen zum Laden von Elektrofahrzeugen (vgl. Dworak 2014; Siemens 2014). Allerdings stellen auch bei diesem Vorschlag die benötigten Netze zur Energiezufuhr den zentralen Engpassfaktor dar, ohne dass sich hierfür bereits eine Lösung abzeichnet. Zudem wird damit nicht die drängende Frage vieler Städte beantwortet, in welche Form von Ladeinfrastruktur (hier: Ladestationen oder Induktionstechnologie) zukünftig investiert werden sollte. Schließlich stellt sich die weitere Frage, ob sich überhaupt ein Ausbau der Ladeinfrastruktur - egal in welcher Variante - lohnt, da unter dem Aspekt der Nutzerakzeptanz gegenwärtig vor allem E-Fahrräder und E-Motorräder durch einen merklichen Nachfrageanstieg gekennzeichnet sind (vgl. Rohwetter 2016). Bleibt es bei diesem Trend, wäre der kapitalintensive Aufbau einer weitgehend flächendeckenden öffentlichen Ladeinfrastruktur nicht erforderlich.

\subsubsection{Elektromobilität im ländlichen Raum}

Nicht zu erwarten ist demgegenüber eine kurz- bis mittelfristig vermehrte Nutzung von E-Fahrzeugen im ländlichen Raum. Dies gilt insbesondere für die agglomerationsfernen (peripheren) ländlichen Räume, da hier die zu überwindenden Distanzen bei der Befriedigung bestehender Mobilitätsbedürfnisse einer größeren Verbreitung elektrischer Antriebe im Wege stehen. Hier dürfte sich - anders als im städtischen Raum - noch am ehesten bei Hybridfahrzeugen (PHEV, REEV) eine größere Nachfrage ergeben. Inwieweit hier die räumliche Ausprägung des demographischen Wandels in Form einer zukünftig zunehmenden Entleerung der ländlichen Räume bei einem gleichzeitigen Bevölkerungszuwachs in den Ballungsräumen zu einer Ausweitung der Nachfrage nach E-Fahrzeugen führen wird, bleibt zum gegenwärtigen Zeitpunkt abzuwarten (vgl. die verschiedenen Beiträge in Mager 2014). Grundsätzlich ist jedoch von einem unterschiedlichen Mobilitätsverhalten in urbanen und ländlichen Räumen auszugehen: „Außerhalb der großen Städte sieht die Welt [...] noch anders aus. Polyzentrische Raumstrukturen und disperse Siedlungsweisen auf der einen und flexible Arbeitszeiten sowie ein über Jahrzehnte gestiegener Freizeitverkehr auf der anderen Seite sind weiterhin dominante Tatbestände. Sie sind auch Ursache dafür, dass Verkehrsströme zerfasern" (Canzler/Knie 2015: 38). Mit Bezug auf die aus psychologischer Sicht betonte habituelle Komponente des Mobilitätsverhaltens lässt sich zudem feststellen, dass gerade im ländlichen Raum die „eingeübte Praxis, das Leben um das eigene Auto herum zu gruppieren, [...] noch über Jahre nach[wirkt]“ (Canzler/ Knie 2015: 13).

Dies führt zu der Einsicht, dass im ländlichen Raum noch mehr als im urbanen Raum der Gebrauch von E-Fahrzeugen einen höheren oder auch anderen Nutzen stiften muss, als dies für konventionelle Fahrzeuge gilt, um eine entsprechende Substitutionsentscheidung auszulösen. Für diese Einschätzung sprechen nicht zuletzt psychologische Studien, wonach - wie bereits an früherer Stelle erwähnt vergangene Verhaltenshäufigkeiten einen starken Einfluss auf das zukünftige Entscheidungsverhalten ausüben und auf diese Weise bei den Nutzern einen „Status quo bias“ erzeugen (vgl. Bamberg 2010; Hunecke 2006). Während jedoch im städtischen Raum ein solcher Zusatz- oder Andersnutzen von E-Fahrzeugen darin gesehen werden kann, Bestandteil einer umfassenderen Energieinfrastruktur sowie Element eines verkehrsmittelübergreifenden Mobilitätssystems (Carsharing, Multimodalität etc.) zu sein (vgl. Bratzel 2015), entfällt dieser Zusatznutzen in der Regel im ländlichen Raum. Soweit E-Fahrzeuge hier eine Alternative darstellen, dürfte dies lediglich für den Ersatz eines vorhandenen Zweitwagens gelten. Zwar wird auch für den ländlichen Raum die Praktikabilität von „Hub-and-Spoke“-Kon- 
zepten als einem möglichen Zusatznutzen von Elektromobilität nicht grundsätzlich ausgeschlossen (vgl. Canzler/Knie 2015). Je peripherer der ländliche Raum jedoch ist, desto unwirtschaftlicher dürfte die Umsetzung eines solchen, die Nutzung von Elektromobilität begünstigenden Konzeptes sein.

\section{Ausblick}

Ob der ,reinen' Elektromobilität (BEV, FCEV) mittel- bis langfristig der Durchbruch gelingt, hängt neben den aufgezeigten verkehrs-, umwelt- und raumbezogenen Implikationen aus entscheidungstheoretischer Sicht sowohl vom technischen Fortschritt vor allem im Bereich der Energiespeichertechnik als auch der (Preis-)Entwicklung auf den relevanten Rohstoff- und Energiemärkten ab, da beide Bestimmungsfaktoren einen entscheidenden Einfluss auf das Kostensenkungspotenzial bzw. die Wirtschaftlichkeit und damit die Nutzerakzeptanz von Elektromobilität haben. Welchen Beitrag staatliche Fördermaßnahmen diesbezüglich leisten können, muss als offen gelten: Eine direkte Subventionierung des Kaufs von Elektrofahrzeugen senkt zwar kurzfristig deren Anschaffungskosten, mindert aber mittel- bis langfristig die Innovationsanreize der Hersteller zur Produktion kostengünstigerer Fahrzeuge. Auch wird in einer solchen Förderung kein geeignetes Mittel gesehen, um die Klimabilanz des Verkehrssektors im Sinne der Nachhaltigkeit zu verbessern. Alternative Maßnahmen wie die Einführung einer einheitlichen Kohlenstoffabgabe oder staatliche Investitionen in alternative Antriebs-, Speicher- oder Kraftstofftechnologien gelten hier als weitaus zielführender (vgl. Dietrich/Leßmann/Steinkraus 2016). Gegen eine staatliche Subventionierung des Kaufs von E-Fahrzeugen spricht zudem, dass unter dem ökologischen Aspekt eine Elektrifizierung des öffentlichen Nahverkehrs (z. B. in Form von 10.000 Hybridbussen) den gleichen Klimanutzen wie die von der Bundesregierung für 2020 formulierte Zielvorgabe von einer Million Elektroautos stiftet (vgl. Asendorpf 2015). Aber auch nichtmonetäre Maßnahmen zur Steigerung der Nutzerakzeptanz sind nicht frei von Problemen, wie beispielsweise das jüngst verabschiedete Gesetz zur Förderung von Elektromobilität, welches unter anderem die freie Nutzung von Busspuren sowie kostenfreie Innenstadtparkplätze für Elektroautos erlaubt. Dies kann im Erfolgsfall sowohl zu einer unerwünschten Begünstigung PSstarker Hybrid-Fahrzeuge (PHEV) gegenüber $\mathrm{CO}_{2}$-armen konventionell angetriebenen Pkw als auch zu Problemen bei der Parkraumbewirtschaftung im urbanen Raum führen (vgl. Lamparter 2014). Von einer ebenso raschen wie flächendeckenden Durchsetzung von E-Fahrzeugen gegenüber konventionellen Antriebstechniken ist damit zumindest für die nähere Zukunft nicht auszugehen.

\section{Literatur}

Ahrend, C.; Delatte, A.; Kettner, S.; Schenk, E.; Schuppan, J. (2014): Multimodale Mobilität ohne eigenes Auto im urbanen Raum. Berlin.

Ahrend, C.; Schwedes, O.; Richter, T. (2011): Teilvorhaben Nutzerverhalten und Raumplanung, Regionale Infrastruktur der Technischen Universität Berlin. Schlussbericht. Berlin.

Asendorpf, D. (2015): Sauber? Kommt drauf an ... Elektroautos sind keinesfalls automatisch umweltfreundlicher als Benziner oder Diesel. In: Die Zeit Nr. 47 vom 19. November 2015, 40.

Bamberg, S. (2010): Alltagsmobilität und Verkehrsmittelwahl. In: Linneweber, V.; Lantermann, E.-D.; Kals, E. (Hrsg.): Spezifische Umwelten und umweltbezogenes Handeln. Göttingen, 549-586. $=$ Enzyklopädie der Psychologie 1 .

Bamberg, S.; Schmidt, P. (2001): Theory-driven subgroup-specific evaluation of an intervention to reduce private car use. In: Journal of Applied Social Psychology 31, 6, 1300-1329.

Bertram, M.; Bongard, S. (2014): Elektromobilität im motorisierten Individualverkehr - Grundlagen, Einflussfaktoren und Wirtschaftlichkeitsvergleich. Wiesbaden.

Bobeth, S.; Matthies, E. (2016): Elektroautos: Top in Norwegen, Flop in Deutschland? Empfehlungen aus Sicht der Umweltpsychologie. In: Gaia 25, 1, 38-48.

Bongard, S. (2014): ECAR-Studie zur Akzeptanz der Elektromobilität. Ludwigshafen.

Boston Consulting Group (2009): The Comeback of the Electric Car? Boston.

Boston Consulting Group (2010): Batteries for Electric Cars - Challenges, Opportunities, and the Outlook to 2020. Boston.

Bozem, K.; Nagl, A.; Rath, V.; Haubrock, A. (2013): Elektromobilität - Kundensicht, Strategien, Geschäftsmodelle. Wiesbaden.

Bratzel, S. (2011): „i-Car“ - die junge Generation und das vernetzte Auto. Eine empirische Studie zu den Einstellungen und Verhaltensmustern der 18-25 Jährigen in Deutschland. Bergisch Gladbach.

Bratzel, S. (2015): Mobilitätschip statt eigenes Auto? In: Die Zeit Nr. 23 vom 3. Juni $2015,33$.

Bundesregierung (2009): Nationaler Entwicklungsplan Elektromobilität der Bundesregierung. Berlin.

Canzler, W.; Knie, A. (2015): Die neue Verkehrswelt. Mobilität im Zeichen des Überflusses: schlau organisiert, effizient, bequem und nachhaltig unterwegs. Berlin.

Degelmann, R. (2014): Elektromobilität - ein Hype-Thema? In: VSVI Bayern - Jahreszeitschrift der Vereinigung der Straßenbau- und Verkehrsingenieure in Bayern e.V. 5, 22-29.

Delbosc, A.; Currie, G. (2014): Changing demographics and young adult driver license decline in Melbourne, Australia (1994-2009). In: Transportation 41, 3, 529-542.

Deutsches Clean Tech Institut (2010): eMobilität - CleanTech-Branche: Treiber im Fokus. Bonn.

Dietrich, A.-M.; Leßmann, C.; Steinkraus, A. (2016): Kaufprämie für Elektroautos - Politik auf dem Irrweg? In: ifo Schnelldienst 69, 11, 21-26.

Döring, T. (2012): Hat die Elektromobilität eine Zukunft? In: Wirtschaftsdienst 92, 8, 563-571.

Döring, T.; Aigner, B. (2011): E-Mobility - Realistic Vision or Hype. An economic analysis. In: Przeglad Elektrotechniczny 87, 3, $37-$ 40.

Döring, T.; Aigner-Walder, B. (2012): Zukunftsperspektiven der Elektromobilität - Treibende Faktoren und Hemmnisse in ökonomischer Sicht. In: Wirtschaft und Gesellschaft 38, 1, 103-132.

Döring, T.; Aigner-Walder, B. (2015): Verkehrs-, umwelt- und raumbezogene Aspekte der Elektromobilität unter Berücksichtigung entscheidungstheoretischer Überlegungen zum Nutzerverhalten. Darmstadt. $=$ sofia-Diskussionsbeiträge zur Institutionenanalyse 15-5. 
Donath, N. (2014): Sound-Design für Elektroautos - Studie zur künstlichen Erzeugung markentypischer Fahrgeräusche. Hamburg.

Dudenhöffer, F. (2014): Pkw-Neuwagen: geringere $\mathrm{CO}_{2}$-Belastungen ohne Zusatzkosten möglich. In: Wirtschaftsdienst 94, 8, 600-602.

Dudenhöffer, F. (2015): Niedrige Treibstoffpreise lassen Neuwagenkäufer in alte Verhaltensmuster zurückfallen. In: Wirtschaftsdienst 95, 8, 548-552.

Dworak, M. (2014): Lampen zu Zapfsäulen. In: Die Zeit Nr. 21 vom 15. Mai 2014, 30.

Egbue, O.; Long, S. (2012): Barriers to widespread adoption of electric vehicles: An analysis of consumer attitudes and perceptions. In: Energy Policy 48, 9, 717-729.

Fazel, L. (2014): Akzeptanz von Elektromobilität - Entwicklung und Validierung eines Modells unter Berücksichtigung der Nutzungsform des Carsharing. Wiesbaden.

FehrAdvice \& Partners (2012): Beurteilung verkehrslenkender Massnahmen beim Einkaufsverkehr unter besonderer Berücksichtigung verhaltensökonomischer Erkenntnisse. Zürich.

Fraunhofer IAO - Fraunhofer-Institut für Arbeitswirtschaft und Organisation; PriceWaterhouseCoopers (2010): Elektromobilität Herausforderungen für Industrie und öffentliche Hand. Frankfurt am Main.

Fraunhofer ISI - Fraunhofer-Institut für System- und Innovationsforschung (2011): Gesellschaftspolitische Fragestellungen der Elektromobilität. Karlsruhe.

Fraunhofer IWES - Fraunhofer-Institut für Windenergie und Energiesystemforschung (2013): Wissenschaftliche Unterstützung bei der Erstellung von fahrzeugbezogenen Analysen zur Netzintegration von Elektrofahrzeugen unter Nutzung erneuerbarer Energien. Kassel/Bremerhaven.

Frick, R.; Belart, B.; Schmied, M.; Grimm, B.; Schmücker, D. (2014): Langstreckenmobilität - Aktuelle Trends und Zukunftsperspektiven. Bern/Kiel.

Fujii, S.; Gärling, T.; Kitamura, R. (2001): Changes in drivers' perceptions and use of public transport during a freeway closure. In: Environment and Behavior 33, 6, 796-808.

Gries, S.; Zelewski, S. (2015): Wirtschaftlichkeit von Elektronutzfahrzeugen. In: Proff, H. (Hrsg.): Entscheidungen beim Übergang in die Elektromobilität. Wiesbaden, 599-615.

Hacker, F.; von Waldenfels, R.; Mottschall, R. (2015): Wirtschaftlichkeit von Elektromobilität in gewerblichen Anwendungen - Betrachtung von Gesamtnutzungskosten, ökonomischen Potenzialen und $\mathrm{CO}_{2}$-Minderung. Berlin.

Haugrund, S. (2013): Elektro-Mobilität wird deutlich überschätzt. In: Wirtschaftsdienst 93, 9, 643-647.

Hidrue, M.; Parsons, G.; Kempton, W.; Gardner, M. (2011): Willingness to pay for electric vehicles and their attributes. In: Resource and Energy Economics 33, 3, 686-705.

Hucko, M. (2013): Wie sicher sind Elektroautos? In: Spiegel Online vom 9. November 2013. http://www.spiegel.de/auto/aktuell/ tesla-braende-experten-glauben-an-sicherheit-von-elektroautosa-932594.html (21.10.2016).

Hunecke, M. (2006): Zwischen Wollen und Müssen. Ansatzpunkte zur Veränderung der Verkehrsmittelnutzung. In: Technikfolgenabschätzung - Theorie und Praxis 15, 3, 31-37.

ifeu - Institut für Energie- und Umweltforschung (2011): UMBReLA Umweltbilanzen Elektromobilität. Ergebnisbericht. Heidelberg.

ifmo - Institut für Mobilitätsforschung (2011): Mobilität junger Menschen im Wandel - multimodaler und weiblicher. München.

InnoZ - Innovationszentrum für Mobilität und gesellschaftlichen Wandel (2014): Machbarkeitsstudie „Klimaneutrales Berlin 2050“. Berlin.

Institut der deutschen Wirtschaft (2014): Infrastruktur zwischen Standortvorteil und Investitionsbedarf. Köln.

Jansen, M.; Richts, C.; Gerhardt, N.; Lenck, T.; Heddrich, M.-L. (2015): Strommarkt-Flexibilisierung. Hemmnisse und Lösungskonzepte. Kassel/Berlin.

Karle, A. (2015): Elektromobilität - Grundlagen und Praxis. München.
Konrad, K.; Wittowsky, D. (2016): Digitalisierung der Lebenswelten junger Menschen - der Zusammenhang von virtueller und physischer Mobilität. Dortmund. = ILS-Trends 1/16.

Korfanty-Schiller, E. M.; Ellermann, S.; Haug, A.; Hirschle, A.; Janetzke, C.; Robaschik, F.; Sauermost, M. (2015): Batterien für Elektroautomobile: Wachstumspläne in Japan, Südkorea, China, den USA und Deutschland. In: Korthauer, R. (Hrsg.): Handbuch Elektromobilität 2015. Frankfurt am Main, 92-110.

Kuhnimhof, T.; Armoogum, J.; Buehler, R.; Dargay, J.; Denstadli, J. M.; Yamamoto, T. (2012): Men Shape a Downward Trend in Car Use among Young Adults - Evidence from Six Industrialized Countries. In: Transport Reviews 32, 6, 761-779.

Kuhnimhof, T.; Buehler, R.; Wirtz, M.; Kalinowska, D. (2012): Travel trends among young adults in Germany: increasing multimodality and declining car use for men. In: Journal of Transport Geography $24,9,443-450$.

Kuhnimhof, T.; Zumkeller, D.; Chlond, B. (2013): Who made peak car, and how? A breakdown of trends over four decades in four countries. In: Transport Reviews 33, 3, 325-342.

Kunert, U.; Horn, M.; Kalinowska, D.; Kloas, J.; Ochmann, R.; Schulz, E. (2008): Mobilität 2025 - Der Einfluss von Einkommen, Mobilitätskosten und Demografie. Berlin.

Kutter, E. (2001): Alltäglicher Verkehrsaufwand zwischen Individualität und sachstruktureller Determination. In: Flade, A.; Bamberg, S. (Hrsg.): Ansätze zur Erklärung und Beeinflussung des Mobilitätsverhaltens. Darmstadt, 205-238.

Kutter, E.; Stein, A. (1998): Minderung des Regionalverkehrs. Chancen von Städtebau und Raumordnung in Ostdeutschland. Bonn.

Lamparter, D. H. (2014): Auf der falschen Spur? In: Die Zeit Nr. 41 vom 1. Oktober 2014, 33.

Lebeau, K.; van Mierlo, J.; Lebeau, P.; Mairesse, O.; Macharis, C. (2012): The market potential for plug-in hybrid and battery electric vehicles in Flanders: A choice-based conjoint analysis. In: Transportation Research Part D: Transport and Environment 17, 8, 592-597.

Löfken, J. O. (2010): E-Mobilität - Die Zukunft rollt auf zwei Rädern. http://www.wissenschaft-aktuell.de/extra_rubriken/E_ Mobilitaet_Die_Zukunft_rollt_auf_zwei_Raedern.html (24.10.2016).

Maaß, C. (2014): Damit es brummt - Eine Pkw-Maut für alle muss her. In: Die Zeit Nr. 37 vom 4. September 2014, 11.

Mager, T.J. (2014) (Hrsg.): Liegt die Zukunft der Elektromobilität im ländlichen Raum? Köln.

McDonald, N. C. (2015): Are Millennials Really the "Go-Nowhere" Generation? In: Journal of the American Planning Association 81, 2, 90-103.

Moons, I.; de Pelsmacker, P. (2012): Emotions as determinants of electric car usage intention. In: Journal of Marketing Management 28, 3-4, 195-237.

NPE - Nationale Plattform Elektromobilität (2014): Fortschrittsbericht 2014 - Bilanz der Marktvorbereitung. Berlin.

Nykvist, B.; Nilsson, M. (2015): Rapidly falling costs of battery packs for electric vehicles. In: Nature Climate Change 5, 329-332.

Papendick, K.; Brennecke, U.; Sánchez Márquez, J. S.; Deml, B. (2011): Nutzerverhalten beim Laden von Elektrofahrzeugen. http://www.iaf-ag. ovgu.de/iniafag_media/downloads/publikationen/ Nutzerverhalten+beim+Laden+von+Elektrofahrzeugen.pdf (24.10.2016).

Paternoga, S.; Pieper, N.; Woisetschläger, D.; Beuscher, G.; Wachalski, T. (2013): Akzeptanz von Elektrofahrzeugen - Aussichtsloses Unterfangen oder große Chance? Wolfsburg/Braunschweig.

Pehnt, M.; Höpfner, U.; Merten, F. (2007): Elektromobilität und erneuerbare Energien. Heidelberg/Wuppertal.

Peters, A.; Doll, C.; Plötz, P.; Sauer, A.; Schade, W.; Thielmann, A.; Wietschel, M.; Zanker, C. (2013): Konzepte der Elektromobilität - Ihre Bedeutung für Wirtschaft, Gesellschaft und Umwelt. Berlin. 
Peters, A.; Hoffmann, J. (2011): Nutzerakzeptanz von Elektromobilität. Karlsruhe.

Pieper, N.; Woisetschläger, D.; Paternoga, S.; Wachalski, T.; Beuscher, G. (2013): Elektromobilität auf dem Prüfstand. Wirksamkeit von Kaufanreizen. Wolfsburg/Braunschweig.

Rohwetter, M. (2016): Läuft bei uns - Elektroautos sind viel zu teuer, aber E-Bikes verkaufen sich prächtig. In: Die Zeit Nr. 52 vom 15. Dezember 2016, 30.

Schönduwe, R.; Bock, B.; Deibel, I. (2012): Alles wie immer, nur irgendwie anders? Trends und Thesen zu veränderten Mobilitätsmustern junger Menschen. Berlin.

Schreckenberg, M. (2015): Mobilität und Mensch: Wie geht es weiter? In: Korthauer, R. (Hrsg.): Handbuch Elektromobilität 2015. Frankfurt am Main, 15-27.

Schwarzer, C. M.; Breitinger, M. (2016): So funktioniert die Kaufprämie für Elektroautos. In: Zeit Online (27. April 2016). http://www.zeit.de/mobilitaet/2016-04/elektroauto-kaufpraemiebestseller (21.10.2016)

Siemens (2014): Elektromobilitätskonzepte für Laternenparker unter Berücksichtigung innerstädtischer Rahmenbedingungen City2.e. München.

Steinhilber, S.; Wells, P.; Thankappan, S. (2013): Socio-technical inertia: Understanding the barriers to electric vehicles. In: Energy Policy 60, 9, 531-539.

Sterner, M.; Eckert, F.; Thema, M.; Bauer, F. (2015): Der positive Beitrag dezentraler Batteriespeicher für eine stabile Stromversorgung. Regensburg.
Teichmann, G.A.; Lehmann, S.; Brandt, T. (2015): Das Klimaschutzziel $2050 \mathrm{im}$ Verkehr - ohne weitere Anreize nicht erreichbar. In: Korthauer, R. (Hrsg.): Handbuch Elektromobilität 2015. Frankfurt am Main, 62-91.

Teufel, D.; Arnold, S.; Bauer, P.; Schwarz, T. (2015): Ökologische Folgen von Elektroautos. Ist die staatliche Förderung von Elektround Hybridautos sinnvoll? Heidelberg. = UPI-Bericht 79.

US Department of Transportation - National Highway Traffic Safety Administration (2009): Incidence of Pedestrian and Bicyclist Crashes by Hybrid Electric Passenger Vehicles. Washington DC.

van Wee, B. (2015): Peak car: The first signs of a shift towards ICTbased activities replacing travel? A discussion paper. In: Transport Policy 42, 8, 1-3.

Wallentowitz, H. (2013): Fokus Batterie - Zur technischen Entwicklung von Elektroautos. In: Keichel, M.; Schwedes, O. (Hrsg.): Das Elektroauto - Mobilität im Umbruch. Wiesbaden, 127-152.

Ziegler, A. (2012): Individual characteristics and stated preferences for alternative energy sources and propulsion technologies in vehicles: A discrete choice analysis for Germany. In: Transportation Research Part A: Policy and Practice 46, 8, 1372-1385.

Ziegler, P.-M. (2011): Elektromobilität - „Weltrekord-Auto“ abgebrannt. In: Heise Online (17. Januar 2011). http://www. heise.de/newsticker/meldung/Elektromobilitaet-WeltrekordAuto-abgebrannt-1170822.html (21.10.2016). 